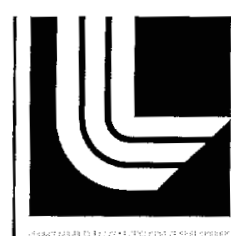

LAWRENCE LIVERMORE NATIONAL: LABORATORY

Laser Science and

Technology Program Update 2002

L. A. Hackel, H. L. Chen

\section{UCRL-ID-134972-02}

\section{March 1, 2003}


This document was prepared as an account of work sponsored by an agency of the United States Government. Neither the United States Government nor the University of California nor any of their employees, makes any warranty, express or implied, or assumes any legal liability or responsibility for the accuracy, completeness, or usefulness of any information, apparatus, product, or process disclosed, or represents that its use would not infringe privately owned rights. Reference herein to any specific commercial product, process, or service by trade name, trademark, manufacturer, or otherwise, does not necessarily constitute or imply its endorsement, recommendation, or favoring by the United States Government or the University of California. The views and opinions of authors expressed herein do not necessarily state or reflect those of the United States Government or the University of California, and shall not be used for advertising or product endorsement purposes.

This work was performed under the auspices of the U.S. Department of Energy by University of California, Lawrence Livermore National Laboratory under Contract W-7405-Eng-48. 


\section{UCRL-ID-134972-02}

\section{Laser Science and \\ Technology Program Update 2002}

L. A. Hackel, H. L. Chen

March 1, 2003 
This document was prepared as an account of work sponsored by an agency of the United States Government. Neither the United States Government nor the University of California nor any of their employees, makes any warranty, express or implied, or assumes any legal liability or responsibility for the accuracy, completeness, or usefulness of any information, apparatus, product, or process disclosed, or represents that its use would not infringe privately owned rights. Reference herein to any specific commercial product, process, or service by trade name, trademark, manufacturer, or otherwise, does not necessarily constitute or imply its endorsement, recommendation, or favoring by the United States Government or the University of California. The views and opinions of authors expressed herein do not necessarily state or reflect those of the United States Government or the University of California, and shall not be used for advertising or product endorsement purposes.

This work was performed under the auspices of the U.S. Department of Energy by University of California, Lawrence Livermore National Laboratory under Contract W-7405-Eng-48. 


\title{
Laser Science and Technology \\ Program Update 2002
}

\author{
L.A. HACKEL AND H.L. CHEN
}

March 2003

Lawrence Livermore National Laboratory University of California $\bullet$ Livermore, California $\bullet 94550$ 


\section{Laser Science and Technology Program}

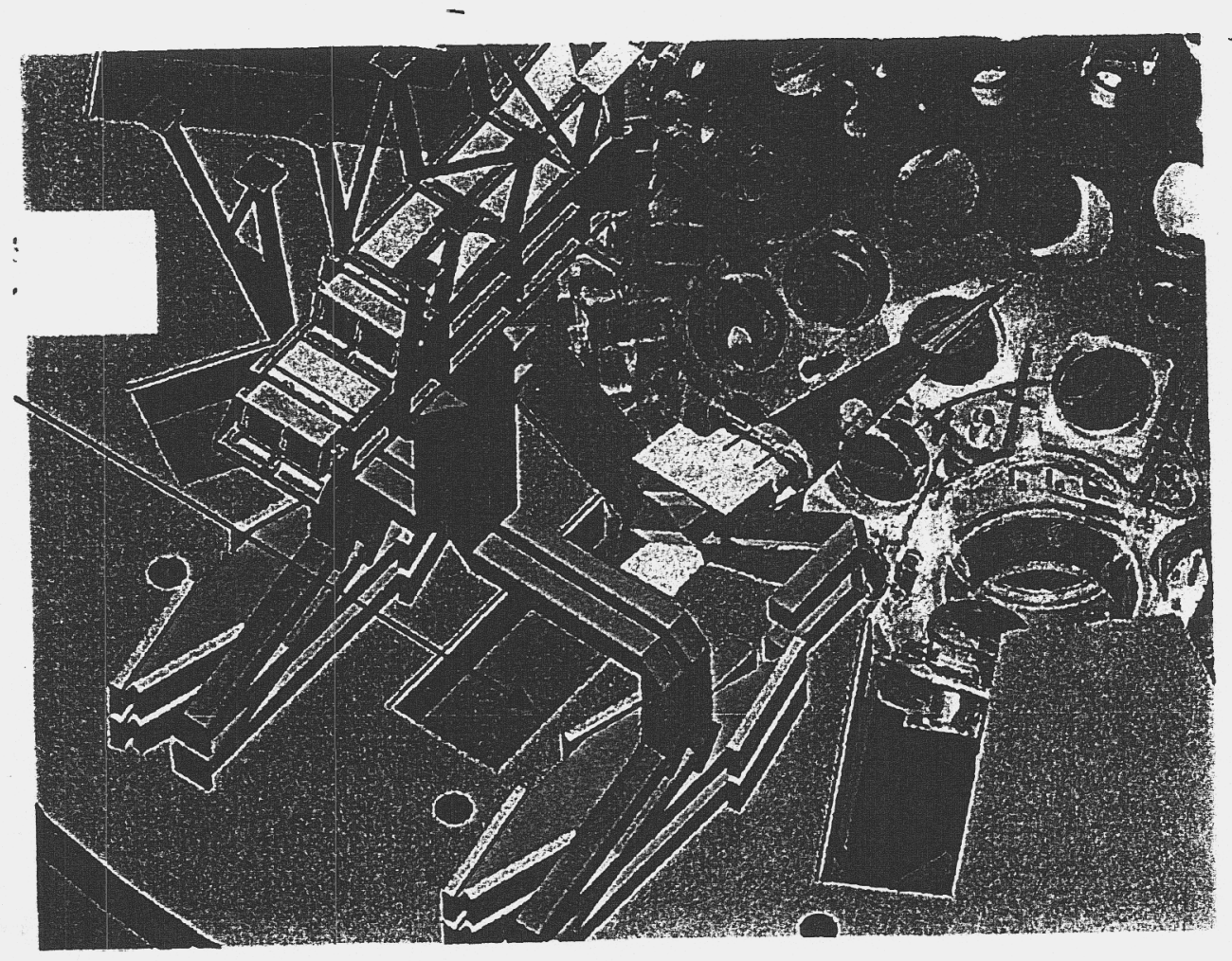

\section{Overview}

The Laser Science and Technology (LS\&T) Program's mission is to develop advanced lasers, optics, materials technologies, and applications to solve problems and create new capabilities of importance to the nation and the Laboratory. A top, near-term priority is to provide technical support in the deployment and upgrade of the National Ignition Facility (NIF). Our other program activities synergistically develop technologies that are of interest to the NIF Directorate but outside the scope of the NIF funding.

The primary objectives of LS\&T activities in 2002 have been fourfold-(a) to support deployment of hardware and to enhance laser and optics performance for NIF, (b) to develop high-energy petawatt laser science and technology for the Department of Energy (DOE),(c) to develop advanced solid-state laser systems and optical components for the Department of Defense (DoD), and to invent develop, and deliver improved concepts and hardware for other government agencies and industry.

Special efforts have been devoted to building and maintaining our capabilities in three technology areas: high-power short-pulse solidstate lasers, high-power optical materials, and applications of advanced lasers. LS\&T activities during 2002 focused on seven major areas:

6. NIF Project-LS\&T led major advances in the deployment of NIF Final Optics Assembly (FOA) and the development of $3 \omega$ optics processing and treatment technologies to enhance NIF's operations and performance capabilities.

2. Stockpile Stewardship Program (SSP)-LS\&T personnel continued development of ultrashort-pulse lasers and high-power, large-aperture optics for applications in SSP, extreme-field science and national defense. To enhance the high- 
energy petawatt (HEPW) capability in NIF, LS\&T continued development of advanced compressor-grating and front-end laser technologies utilizing optical-parametric chirped-pulse amplification (OPCPA).

3. High-energy-density physics and inertial fusion energy-LS\&T continued development of $\mathrm{kW}$-to-MW-class, diodepumped, solid-state laser (DPSSL).

4. Department of Defense (DoD)_-LS\&T continued development of a $100 \mathrm{~kW}$-class solid-state heat-capacity laser (SSHCL) for missile defense.

5. Nuclear energy applications-LS\&T continued to develop laser-shock peening technology to improve the service lifetime of metal canisters designed for DOE's Yucca Mountain Project.

6. Materials processing--Under cooperative research and development agreements (CRADA) with U.S. industry, LS\&T developed and delivered $\mathrm{kW}$-class solidstate lasers for shock peening and hole drilling of metals.

7. Diffractive optics for space telescopes and petawatt Lasers-LS\&T continued fabrication of large aperture beam sampling gratings (BSGs) for NIF, and development of large-scale, lightweight diffractive optics for the next generation of space telescope (Eyeglass).

\section{Improving NIF Laser Optics and Material Performance}

LS\&T work on NIF has achieved major strides leading to the deployment of NIF Final Optics Assembly (FOA) and the development of $3 \omega$ optics processing, treatment, and testing technologies to enhance the performance of optics in the FOA. To extend optics lifetimes in the laser chain, we have successfully developed detailed optics processing and treatment procedures. We have completed construction of a preproduction facility (named Phoenix) for conditioning and repairing surface damage on full-scale NIF optics using ultraviolet (UV) and infrared (IR) lasers. We have also upgraded the Optical Sciences Laser (OSL) facility to $1.5 \mathrm{~kJ}$ per pulse to perform optical damage tests on $3 \omega$ optics for NIF. Ten large-aperture beamsampling gratings (BSGs) have already been fabricated and four were installed on NIF. We are installing and commissioning a precision diagnostic system (PDS) for the characterization and commissioning of the beamlines during NIF's early light (NEL). We are also improving advanced optical fabrication technologies to fabricate continuous phase plates (CPP) for NIF.

(P. Wegner)

\section{Enhanced Optical Damage Resistance of NIF Optics Using UV and IR Lasers}

The output fluences that can be attained on NIF and other megajoule-class solid-state lasers are ultimately limited by laser-induced damage to optical components within the chain. When damage reaches performance-limiting levels, optics must be replaced leading to higher operating expense and longer laser downtime. Because the laser damage threshold of optics decreases with decreasing laser wavelength, the final optics operating at 527 and $351 \mathrm{~nm}$ are the most susceptible to damage. The final optics on the NIF consist of frequency-doubling and tripling crystals fabricated from potassium dihydrogen phosphate (KDP) and deuterated $\mathrm{KDP}$, final focusing lens, $\mathrm{BSG}$, and debris shield fabricated from fused silica. Over the past years, we have developed optic treatment techniques using scanning UV laser beams to condition fused silica optics to increase their damage threshold. We have also demonstrated that $\mathrm{CO}_{2}$ lasers can be used to stabilize damage sites on optics and control the size of damage sites to less than few tens of microns, thereby greatly increasing the lifetime of an optic through reuse. With activation of the NIF proceeding, the ability to process full-scale $43-\mathrm{cm} \times 43-\mathrm{cm}$ optics is timely. We have successfully activated Phoenix to condition and repair surface damage on full-scale NIF optics. This new facility is equipped with two UV conditioning lasers-a 50-Hz Nd:YAG laser operating at $355 \mathrm{~nm}$, with a pulse width of $3.7 \mathrm{~ns}$ and a $100-\mathrm{Hz} \mathrm{XeF}$ excimer laser operating at $351 \mathrm{~nm}$ with $23-\mathrm{ns}$ pulse width. The facility also includes a $\mathrm{CO}_{2}$ laser for damage mitigation, an optics stage for raster scanning full-scale optics, two microscopes to image damage sites with $\approx 5-\mu \mathrm{m}$ resolution, and a damage mapping system (DMS) that can detect damage sites or precursors as small as $\approx 15 \mu \mathrm{m}$ to image full-scale optics. The optics are handled in a class 100 clean room within the facility that is maintained at better than class 10000 . 


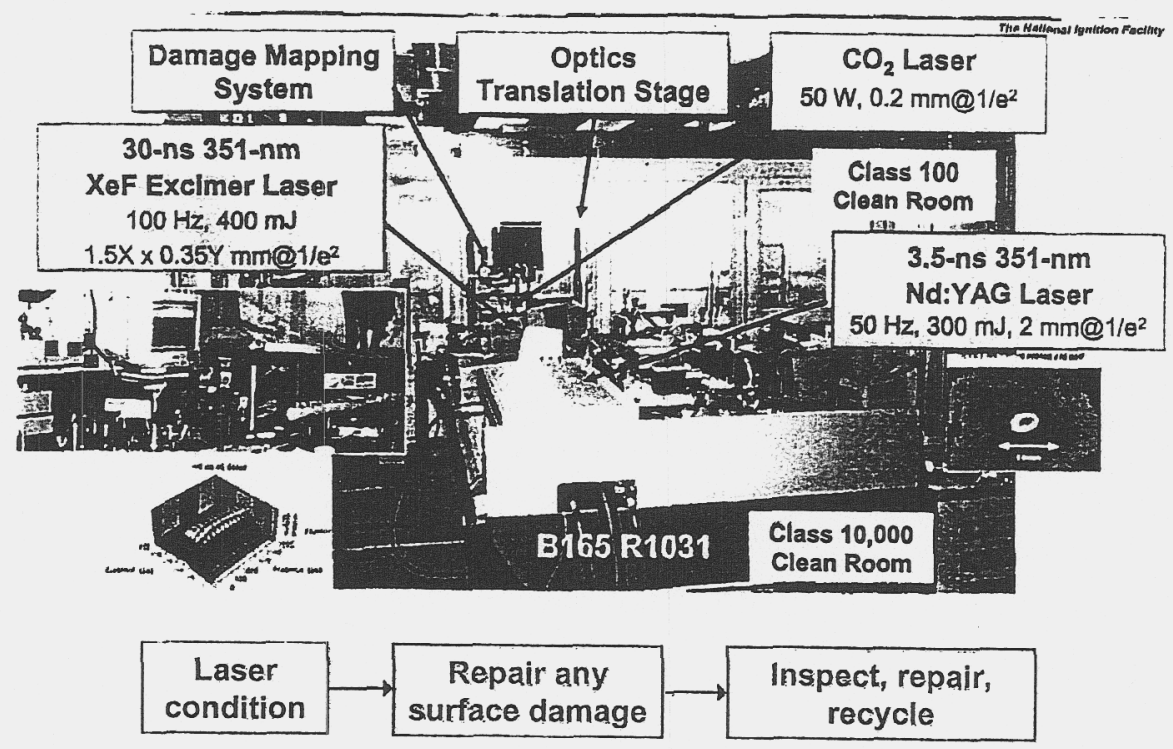

Three full-scale NIF optics were conditioned in Phoenix, including two wedged final focus lenses fabricated from inclusion-free fused silica. The $\mathrm{XeF}$ excimer laser puts out 280 $\mathrm{mJ} / \mathrm{pulse}$. It uses a one-dimensional beam homogenizer to tailor the beam profile that converts the 24-mm $\times 12-\mathrm{mm}$ beam from the laser into a $1.5-\mathrm{mm}$ by $0.3-\mathrm{mm}$ focal spot. Due to the relatively small f-number of the optical system (f/13), the beam cannot be focused at both front and rear surfaces of the optic simultaneously. We thus condition the optic in two steps: output surface first and then the input surface. The first scan is performed at $11 \mathrm{~J} / \mathrm{cm}^{2}$ (which is equivalent to a fluence of $4 \mathrm{~J} / \mathrm{cm}^{2}$ for 3ns pulses, using $\tau^{0.5}$ scaling). A damage map is acquired to identify new sites or sites that have grown in size. These sites are then spot-treated with the $\mathrm{CO}_{2}$ laser to eliminate their potential for damage growth. The UV laser treatment process is repeated at 6,8 , and $10 \mathrm{~J} / \mathrm{cm}^{2}$ (all in 3-ns equivalent) for an optic to be used at $6 \mathrm{~J} / \mathrm{cm}^{2}$ and further at 12 and $14 \mathrm{~J} / \mathrm{cm}^{2}$ for an optic destined for $8 \mathrm{~J} / \mathrm{cm}^{2}$ operation. Using UV laser conditioning and IR laser annealing processes, we have been able to maintain $<1$ defect (or damage site) per fused silica optic at $14 \mathrm{~J} / \mathrm{cm}^{2}$, a $100 \times$ improvement compared to last year's result obtained from subscale optics processed in Phoenix. The design goal for $3 \omega$ optics is 50 defects per optic. We are designing a production facility to condition and mitigate damage on NIF optics. This facility will be commissioned in 2004.

(R. Prasad)
Qualification of $3 \omega$ Optics Using Slab and OSL Lasers

LS\&T has established two laser facilities to test FOA optics and certify finishing and post-treatment processes. Optical damage tests on small optics are typically performed using a small laser beam ( $<1 \mathrm{~mm}$ diameter, Gaussian) to raster over the surface of the optics at high repetition rate. To test NIF optics that have relatively large apertures $(40 \times 40 \mathrm{~cm})$, a large-area, high-energy and high-intensity laser beam that can cover many square millimeters in one shot is required. The OSL and Slab laser facilities were modified to provide such largearea, high-power optical testing capability. The OSL's 10-cm disk amplifier system delivers up to $12 \mathrm{~J} / \mathrm{cm}^{2}$ of $0.351-\mu \mathrm{m}, 3-\mathrm{ns}$ laser light in a round $3-\mathrm{cm}$ diameter, flat-top beam at a repetition rate of 2 shots/hour. The flexible pulse shaping and excellent beam quality of this laser make it uniquely suitable for simulating NIF laser conditions on subscale parts of up to $15 \mathrm{~cm}$ in size. A main focus during this past year has been the characterization and quantification of surface damage on diamond-turned deuterated potassium dihydrogen phosphate (DKDP). Extensive testing of $15-\mathrm{cm}$ material was performed to correlate surface damage to specific defects on the surface of the DKDP (incurred during the diamond finishing process). The finishing protocol has since been refined to effectively eliminate damage due to these defects. Other work included validation of damage mitigation and stabilization techniques applied to DKDP and fused silica surfaces, validation of small-beam laser conditioning protocols for surface and bulk damage, and the testing of fused silica contaminated with NIF- 


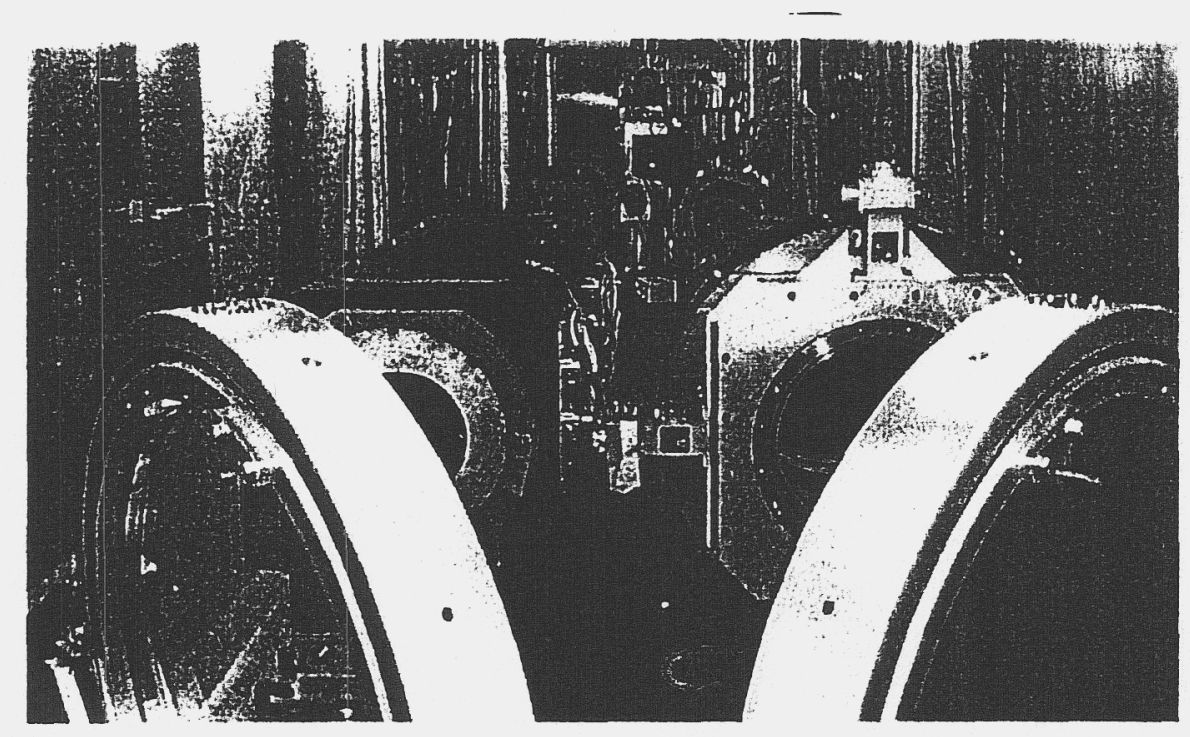

like shrapnel and target debris for establishing debris shield lifetime.

In 2002, LS\&T upgraded the OSL laser to generate $1.5 \mathrm{~kJ}$ of $1053-\mathrm{nm}$ light for testing of NIF final optics at full scale. OSL Upgrade uses a double-pass architecture containing four $20-\mathrm{cm}$ aperture disk amplifiers (twelve disks total). The first-article Integrated Optics Module (IOM) has been installed and experiments are underway to verify the frequency converter operation and to test performance at various $3 \omega$ fluence levels.

The Slab laser can deliver up to 12 $\mathrm{J} / \mathrm{cm}^{2}$ of $0.351-\mu \mathrm{m}, 11-\mathrm{ns}$ laser light in a square 5 -mm flat-top beam at a repetition rate of 30 shots/minute. The repetition rate of this laser enables a NIF lifetime-of-shots to be applied to a test sample in less than a day. Recent work has focused on quantifying the damage growth rate on fused silica surfaces at the individual and combined harmonic wavelengths of Nd:glass, and validating damage mitigation techniques for DKDP surfaces. Current techniques for mitigating damage on the surface of DKDP involve the use of a high-speed diamond-tipped rotary tool to excavate the damage site. The Slab facility has also played an important role in certifying "non-optical" materials for $1 \omega$ and $3 \omega$ laser exposure. Many engineering applications on the NIF require the use of materials able to withstand diffuse or scattered laser fluences of up to a few joules $/ \mathrm{cm}^{2}$ without generating particles or volatile by-products that can put nearby optical components at risk. Slab experiments were instrumental in providing NIF engineers with a plastic alternative to absorbing glass for protecting beam transport system from stray $l \omega$ light at fluences up to $1 \mathrm{~J} / \mathrm{cm}^{2}$.

(M. Nostrand, P. Wegner)
Installation and Activation of the Precision Diagnostic System (PDS) on NIF

LS\&T personnel are also

commissioning an optical Precision Diagnostic System (PDS) to characterize NIF output beams during the NEL campaign. This system makes use of the $1 \omega$ and $3 \omega$ diagnostics from Beamlet, where they were used to demonstrate that the NIF laser architecture could produce the $3 \omega$ energy and far-field beam quality needed for its missions. The PDS will provide a full complement of $1 \omega$ and $3 \omega$ diagnostics and make possible the first full-power and full-aperture test of an Individual Optics Module (IOM) - the optical system that mounts directly to the target: chamber for each beamline and contains the vacuum window, harmonic conversion crystals, focus lens, diagnostic grating, wave plates, and debris shields. The major components of the PDS consist of turning mirrors that transport the laser beam from the output of the tranisport spatial filter to the PDS, a roving mirror diagnostic assembly (RMDA) that contains remotely operated robotic handlers and mirrors, a prime focus vessel (PFV), and a target diagnostic chamber (TDC). The TDC is an 8-foot-diameter by 40 -foot-long vacuum vessel that contains the beam splitters and down-conversion optics. The entire RMDA enclosure and the beamline to the IOM is sealed and filled with Ar to suppress stimulated rotational Raman scattering during high-power laser shots in PDS. Full activation of the RMDA will allow any one of the 96 beamlines in NIF Switchyard 2 to be transported and analyzed. Commissioning of NIF beamlines using PDS will start in 2003.

(J. Murray) 


\section{High-Energy Petawatt Laser Sciences and Technologies}

\author{
Development of chirped pulse \\ amplification has made possible production of \\ unprecedented peak power from lasers. With \\ NIF scheduled to produce first light in 2003, \\ there is an increasing interest in the scientific \\ potential of high-energy petawatt (HEPW) pulse \\ production at NIF and other high-energy laser \\ facilities in the U.S. A high-energy petawatt \\ laser initiative has recently been initiated by the \\ DOE to evaluate this frontier technology. LS\&T \\ has accomplished a number of major milestones \\ in the development of ultrashort-pulse lasers and \\ high-power, large-aperture optics for \\ applications in SSP, extreme-field science, and \\ national defense. \\ (M. Hermann)
}

\section{Petawatt Science and Technology for DOE}

With support from the LDRD Program and the DOE, we are leading an effort to develop a multi-kJ HEPW laser on NIF. The addition of HEPW to NIF will enable the acceleration of data collection for the Stockpile Stewardship Program and new high-energy-density science (HEDS) experiments for advanced inertial confinement fusion (ICF). Several NIF missions benefiting from HEPW capability have been evaluated. These experiments include: fast ignition, high photon energy $(10-\mathrm{keV}$ to $>10-$ $\mathrm{MeV}$ x-ray) backlighters for large, massive targets, unique Equation of State (EOS) studies, 10-to-50 MeV proton backlighting for electric and magnetic field measurements, ultrahot hohlraums for spectral opacity studies and isochoric heating of inertially confined matter using either the relativistic electrons or ballistically focused protons. Our near-term goal is to develop a 2- to 5-kJ, 5- to 50-ps petawatt laser source for performing HEDS experiments with irradiance in the $10^{19}$-to- $10^{20} \mathrm{~W} / \mathrm{cm}^{2}$ range. Our effort in 2002 has focused mainly on designing of advanced laser and optics technologies required to field HEPW beamlines on NIF using existing beamline design for nspulse operation. A conceptual design for installing HEPW capability on NIF was completed. To achieve this goal, some modifications to NIF's injection laser system and target areas will be made.

LS\&T is developing a stable and correctly phased seed laser as the front end of the
HEPW system. We plan to add a short-pulse oscillator and stretcher to the NIF master oscillator room. Output from the oscillator is delivered to a short-pulse preamplifier module to provide a high-contrast chirped pulse for injection to the main NIF amplifier chain. The short-pulse preamplifier module is a line replaceable unit with the capacity to convert from 1 to 4 beams in a NIF quad to short-pulse operation. Dispersion compensation is also added in the preamplifier beam transport system. to allow high-order phase adjustment on individual beamlines. To enhance the performance of the Nd-glass laser amplifier, an efficient broadband preamplifier technology utilizing optical parametric chirped pulse amplification (OPCPA) was developed and evaluated as a possible front end for the HEPW. We are also constructing a compact, ultrahighstability, 100-fs mode-locked fiber laser as master oscillator. A large-magnitude, chirped fiber Bragg grating was designed and procured to stretch the seed pulses to multi-nanoseconds. A large-flat-mode (LMF), high-energy (>1-mJ) fiber preamplifier is also being evaluated.

To enable HEPW on NIF, LS\&T is also developing high-energy, short-pulse compatible gratings and focusing optics for the back-end system. Four gratings would be used to compensate for both temporal and spatial chirp during pulse compression. Since the laser pulse is shortest and energy is highest on the final grating and focusing optic, optical damage of these components is a potential problem. Major effort was focused on developing high-damageresistance, large-aperture multilayer dielectric reflection gratings for compression of the amplified pulses. Initial tests indicate that multilayer dielectric (MLD) coated gratings made with a fused silica $\left(\mathrm{SiO}_{2}\right)$ top layer hold promise for high-energy ( $\sim 5 \mathrm{~kJ})$ operation of a NIF HEPW. The reflection grating developed for HEPW was made with a multilayer stack of dielectric oxides that provide reflectivity at wavelength and angle of use and a $\mathrm{SiO}_{2}$ grating layer with width and height of grooves designed for maximum efficiency and lowest electric field structure at a specific incidence angle. Using photoresist and holographic exposure tools and a large-area ion beam etcher, we were able to generate the grating grooves with high precision. The key to high-damage-fluence MLD gratings is to minimize the E-field enhancement at the groove surface. We are currently fabricating and damage testing sample gratings compatible with $\mathrm{kJ}$-level short pulse production. Our goal is to 
build 2-meter aperture grating structure with $75 \%$ compressor efficiency and individual grating efficiency and parabolic focusing optics is shown at the $>94 \%$. A schematic of the pulse compressors beginning of this report.

(C. Barty and M. Hermann)

\section{Generation of X-Rays by Thomson} Scattering between an Ultrashort Laser Pulse and a Picosecond Electron

\section{Bunch}

With funding from the LDRD Program and in partnership with the Physics and Advanced Technologies (PAT) and the Engineering Directorates, LS\&T continues to develop an ultrafast $x$-ray source based on Thomson scattering of fs laser pulses from relativistic-electron bunches from a linear. accelerator (linac). After years of intensive $R \& D$, the experimental setup for Thomson scattering is returning results. In recent experiments, we successfully generated $70-\mathrm{keV}$ $\mathrm{x}$-rays with extremely high brightness. The immediate application of this intense x-ray source (20-100 keV) will be in pump-probe experiments, which for the Stockpile Stewardship Program to temporally resolve structural dynamics and atomic motion in high- $Z$ materials with ps-resolution.

The PLEIADES facility (Picosecond

Laser Electron InterAction for Dynamic

Evaluation of Structures) is located in

Bldg.194 where PAT's high current, rf, electron linac and LS\&T's fs-laser facility (FALCON, fentosecond accelerator laser concept) are installed. We have recently completed construction and synchronization of a dual-beam Ti-sapphire laser system for photon-electron counter-propagation experiments. A single, mode-locked oscillator is used to seed both the main IR laser system (the source of fs photons) and the photoinjector laser system (PLS, which seeds the linac). The laser master oscillator is phase-locked to an rf crystal oscillator to drive the klystrons that power the linac. The PLS consists of a regenerative amplifier (regen) and single 4-pass amplifier that yields an IR output of $\sim 100 \mathrm{~mJ}$. The amplifier output pulse is compressed using a small, dual grating compressor. The compressor output pulse is frequency-tripled to $266 \mathrm{~nm}$ and transported to the photogun to produce photoelectrons from a polished copper cathode. The photoelectrons are accelerated to $5 \mathrm{MeV}$ and injected into the linac and accelerated between $50-$ to- $80 \mathrm{MeV}$.

In the main IR laser, after the grating. stretcher, the 600-ps pulse is amplified to $1 \mathrm{~J}$ by three stages: a regen, a 4-pass-preamplifier and a second 4-pass power amplifier; and then transported to a vacuum grating compressor. The 600-ps, 1-J pulse is compressed to $~ 50 \mathrm{fs}$ and propagated (in vacuum) to the interaction region. Inside the interaction chamber, the electron pulse is focused by a magnetic lens to a $50-\mu \mathrm{m}$ spot. The laser pulse is delivered and focused to the same interaction point from the opposite direction to collide with the electron bunch at an $\sim 180^{\circ}$ angle.

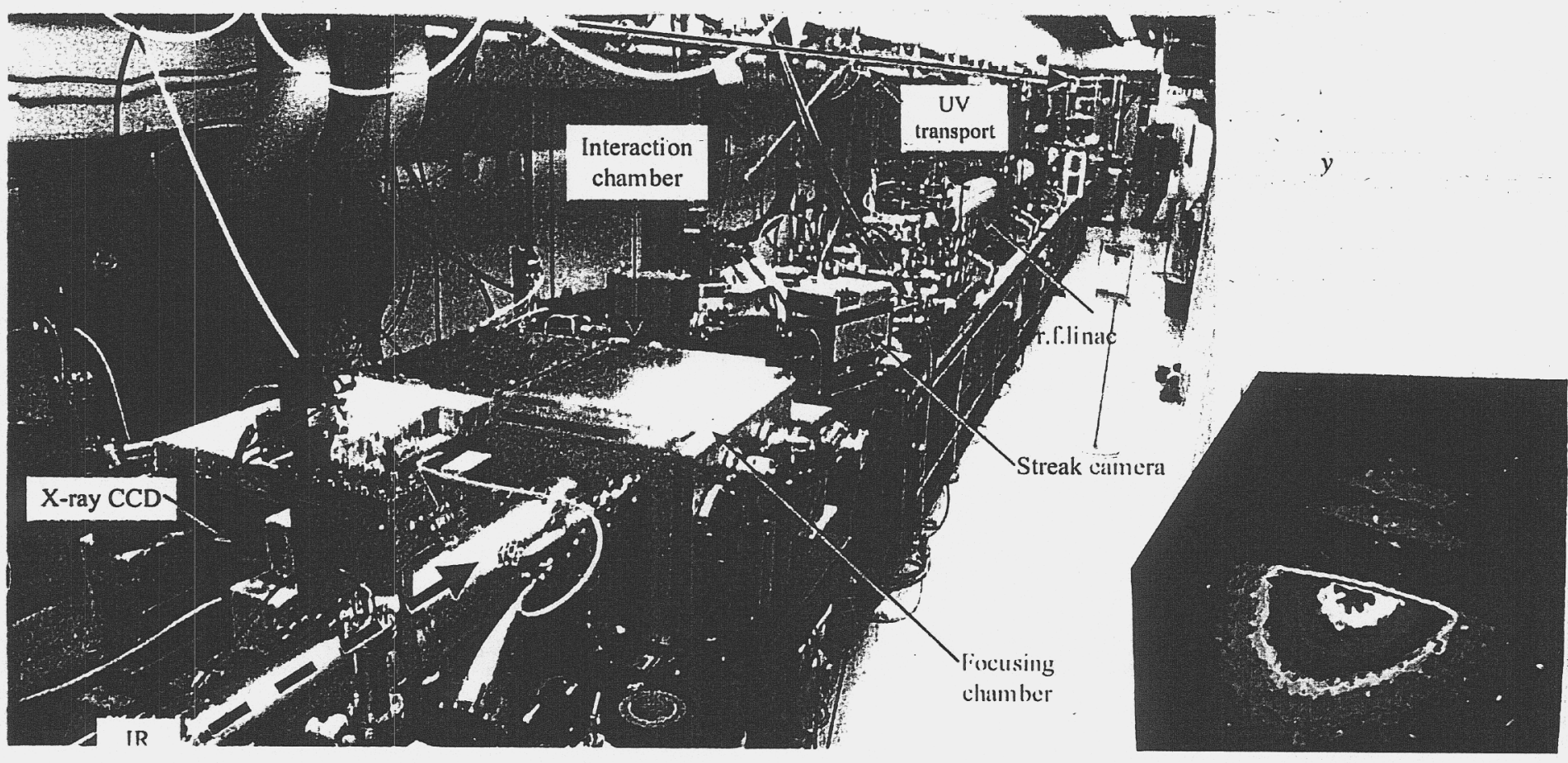


In the scattering chamber, the laser photon energy, $E_{\mathrm{L}}$, is upshifted by an amount proportional to the square of the relativistic energy of the electrons. In our current experimental arrangement, the electron energy is $54 \mathrm{MeV}$, the energy of $800-\mathrm{nm}$ photons $\left(E_{\mathrm{L}}\right)$ is $1.55 \mathrm{eV}$, and so the energy of x-ray photons $\left(E_{\mathrm{x}}\right)$ is calculated to be $70 \mathrm{keV}$. Initial experiments were performed with the laser pulse and electrons interacting head-on. We are also designing experiments for $90^{\circ}$ scattering between the electrons and photons, which will produce sub-picosecond $\mathrm{x}$-ray pulses.

Several diagnostic tools were developed to monitor the Thomson $x$-rays. The image of the Thomson $x$-ray beam monitored by a $x$-ray charge-coupled device (ccd) camera was also recorded. The top portion of the beam was occluded by one of the $x$-ray photodiode packages. The estimated $\mathrm{x}$-ray flux for this shot was $10^{5}$ photons at an average energy of $70 \mathrm{keV}$. This is the highest single pulse ps $\mathrm{x}$-ray flux ever produced at this exergy. Two other groups, one at LBL and the other in Japan, have produced comparable $x$-ray fluxes at lower energy, $30 \mathrm{keV}$ and $14 \mathrm{keV}$ respectively. With straightforward improvements to the laser focal fluence and focused electron spot, we expect an $\sim 1000$ fold improvement in the $\mathrm{x}$-ray flux in future experiments.

(J.Crane, C. Barty)

\section{Short-Pulse, High-Average-Power Laser System for Micromachining}

Under a work-for-others contract, LS\&T is developing a 100-W class picosecond, $\mathbf{k H z}$, solid-state laser system for rapid, precision drilling of small ( $\mu \mathrm{m}$-scale) holes in metal and alloys. We have recently completed the assembly and initial testing of this advanced laser drilling system and were able to deliver over $35 \mathrm{~W}$ in a 2-ps, 4-kHz pulse train to the workpiece for material processing application.

This short-pulse, high-average-power laser employs numerous advanced technologies of the LS\&T program. These technologies include fiber mode-locked oscillators, solid-state regenerative and power chirped-pulse amplifiers using diode-pumped $\mathrm{Yb}: \mathrm{YAG}$ as the gain medium, and a high-power multilayer dielectric grating. The laser system architecture is based on chirped pulse amplification (CPA). It starts with a mode-locked $\mathrm{Yb}$-doped fiber laser, which was developed by Imra Corporation specifically for this application. This mode-locked oscillator produces 8-psec pulse trains and has an average power of $120 \mathrm{~mW}(2.4 \mathrm{~nJ} /$ pulse at $50 \mathrm{MHz})$. The spectral bandwidth of the oscillator pulses is 2 $\mathrm{nm}$. The psec pulses from the fiber oscillator are stretched in time to 2 nsec using a diffraction grating pulse stretcher, amplified by $90 \mathrm{~dB}$, then recompressed to 2-psec duration before being sent to the target chamber. Between each of these laser subsystems, we use computer-based pointing and centering loops to precisely control laser beam alignment.

The stretching-and-compressing process that is the crux of the CPA architecture is accomplished using a high-efficiency dielectric diffraction grating manufactured by LS\&T's Diffractive Optics Group. This large $(150 \times 335$ $\mathrm{mm}^{2}$ ) multi-layer dielectric grating has a diffraction efficiency of $>97 \%$ at $1030 \mathrm{~nm}$. Whereas a typical CPA-based laser system architecture may use 4 diffraction gratings ( 2 for the stretcher and 2 for the compressor), the current design employs a single grating that is shared by both the stretcher and compressor. This single-grating stretcher/compressor design enabled us to combine two of the laser subsystems in a compact unit that can readily be incorporated into a hardened system that can withstand the harsh conditions typically encountered on the factory floor. The $90 \mathrm{~dB}$ of amplification between the pulse stretcher and compressor is accomplished by two subsystems:
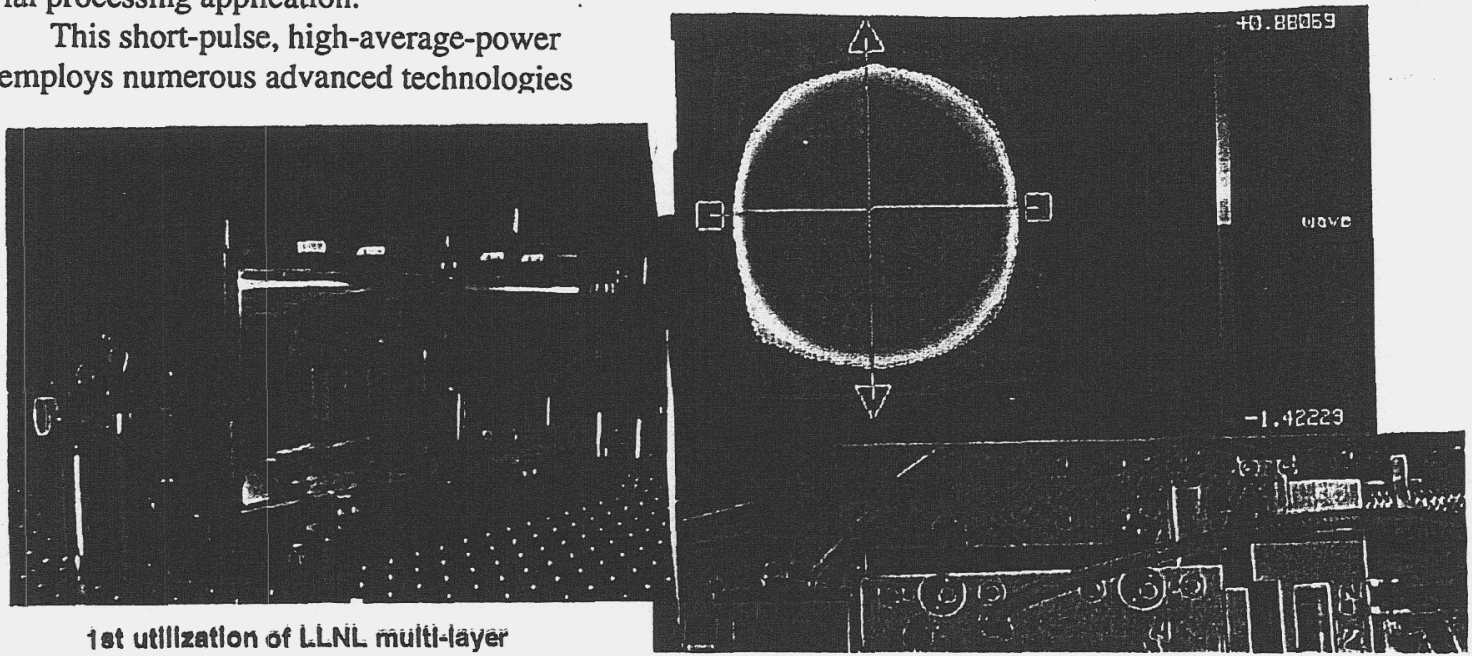
a linear-cavity regenerative amplifier and a power amplifier. Both amplifiers use Yb-YAG as the gain medium, and the power amplifier is pumped with microchannel-cooled diode laser arrays (operated at $940 \mathrm{~nm}$ ). The regen amplifies the $1 \mathrm{~nJ}$ stretched-pulses up to $750 \mu \mathrm{J}$ and converts the pulsing frequency from $50 \mathrm{MHz}$ to $4 \mathrm{kHz}$ (using a Pockels cell). The power amplifier has two heads. It boosts the regen output from $3-W$ to $50-W$ in two passes. With the use of a grating compressor, the pulse width of the amplified beam from the power amplifier is compressed from 2 ns back to 2 psec, multiplying the laser peak power by a factor of a thousand.

For drilling or material processing, the output from the compressor is delivered directly to the workpiece in the target chamber. Online beam diagnostics are developed to record and control the output power, beam quality, and wavefront of the laser. We have made good progress in the drilling of sub- $\mu \mathrm{m}$ holes in metals and alloys as required by our industrial partner. Under the support of ICF's Target Science and Technologies group, we have also built a new cutting station and coupled to an existing shortpulse machining laser to drill high-aspect-ratio, micron-scale holes for fusion target fabrication. Early trials of the system have demonstrated the feasibility of drilling holes that extend more than 100 times beyond the system's Rayleigh range. Holes with 3- $\mu \mathrm{m}$ diameter have been drilled in $125-\mu \mathrm{m}$-thick $\mathrm{Be}$ and $\mathrm{Al}$ foils as well as thinwalled $\mathrm{Be}$ capsules.

(J.Crane, B. Stuart)

\section{Fabrication of Beam Sampling Gratings (BSGs) for Power Balancing of NIF Laser Beams}

Balancing the power on all the NIF beams requires a precise power measurement on each individual beam. This measurement has to be stable to $\pm 1 \%$ over a period of time and should be directly relatable to the energy on target without additional ambiguities or uncertainties. This requires that the measurement be done as close to the end of the laser chain as possible. Using a Fresnel reflection off an optical surface does not provide the required stability because the antireflection coatings degrade over a period of time, and because a reflected sample beam does not travel through all of the optical surfaces. A transmissive diffraction grating inserted in the beam path provides a stable sample of the beam for the power measurements. The requirements on this beam sampling grating

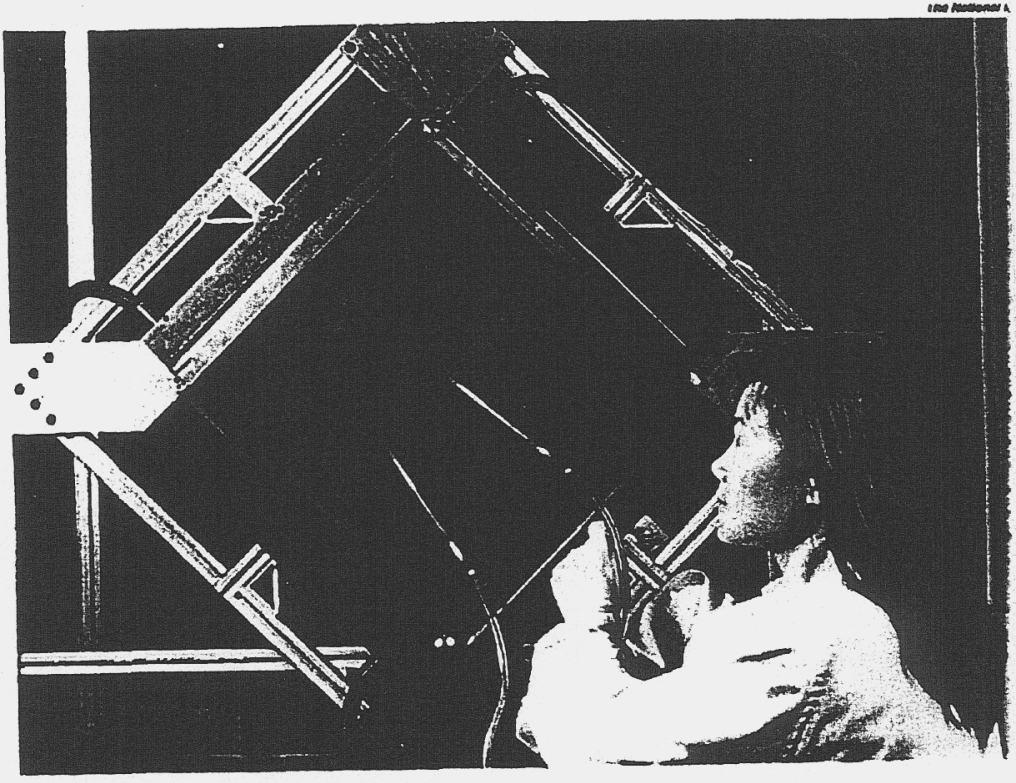

for NIF are that the grating diffract in rransmission a small fraction $(-0.3 \%)$ of the incldent high-power laser beam and bring it to focus at a distance of approximately $1.2 \mathrm{~m}$ from the optic. This requires a shallow grating structure ( 15 to $20 \mathrm{~nm}$ deep) with concentric grooves. The grating period ranges from 1 to 3 $\mu \mathrm{m}$. LS\&T personnel assembled a wet-chemical processing machine and a patterning-andillumination station to fabricate full-size BSGs with 5- to 30-nm groove depth for power balance diagnostics on NIF. We have completed building and testing of production hardware for fabricating BSGs. Ten full-aperture fused silica BSGs have been fabricated for use on the NIF to be used on NIF early light campaign.

$$
\text { (J. Yu, J.Britten) }
$$

\section{Development of Continuous Phase Plates for NIF with Wet-Etch Figuring}

With support from the Laboratory Directed Research and Development (LDRD) Program, we continued to develop a low-cost wet-etching tool for precision optical figuring and finishing of full-aperture optics for NIF. Wet-etch figuring (WEF), is a method for generating arbitrarily shaped optical surfaces using wet chemical etching. During the fabrication process, the etching region on the optic is confined to a stable droplet size through the use of surface tension gradients (called Marangoni confinement), and is moved in a controlled fashion over the optic surface to precisely generate the desired optical figure on very thin glass substrates. Real-time measurement of the surface wavefront during the etching process and feedback allows for a closeloop figuring operation. Over the course of this year, we further refined the process and applied 
it to fabricate subscale continuous phase plates (CPPs) for testing. A small-aperture (30- $\times 30$ $\mathrm{mm}$ ) CPP was fabricated on 1-mm-thick borosilicate substrates. This optic is designed as a beam homogenizer to generate a smooth, super-Gaussian focal spot at target. The surface height gradients for this optic are $0.5 \mu \mathrm{m} / \mathrm{mm}$. Our ultimate goal is to produce full-aperture (40$\mathrm{cm} \times 40-\mathrm{cm}$ ) CPPs for NIF in the future. For precision material processing using short-pulse lasers, we have also fabricated ultrathin (1-mm) phase plates for modifying the focal spot on the workpiece.

(M. Rushford, S. Dixit, J. Britten)

\section{Fabrication of a 5-m-Diameter Diffractive Fresnel Lens for Space-Based Applications}

Detection and imaging of distant objects, such as the exosolar (outside our solar system) planets, require optical telescopes with large-aperture primary optics. Large apertures gather more light from these objects, thereby increasing the signal-to-noise ratio. Space deployment of such telescopes severely limits the weight of the optical components and requires that the primary optic be thin, lightweight, and compact for deployment. Transmissive optics (lenses) are less sensitive to surface ripples and pointing errors than reflective optics (mirrors). Lenses can be made lightweight by replacing conventional lenses with diffractive Fresnel lenses.
Funded by LDRD, LS\&T continues to develop large-aperture Fresnel lenses for space telescopes (Eyeglass project). In an early effort, we built diffractive telescopes at apertures up to $20 \mathrm{~cm}$ diameter and demonstrated their colorcorrected operation over a 470- to 700 -nm-wide bandwidth. We also successfully built largeaperture, space-deployable optics that can be packaged into a small volume for launch. For our first assembly, we built a 75-cm-diameter Fresnel lens made up of six segments and assembled it using $2.5-\mathrm{cm}$-wide metal hinges. After fabrication, this lens and was shown to produce a diffraction-limited focal spot unaffected by folding and unfolding operations.

To further demonstrate the feasibility of scaling this concept to larger sizes, we have recently built and operated a 5-m-diameter Fresnel lens. This lens consists of 72 segments of 0.7-mm-thick glass panels that are glued together using 5-cm-wide metal hinges. The particular layout of the pattern was chosen to allow the lens to be folded into a cylinder of $\sim 1 / 3$ its diameter. The glass panels used for this lens are mass produced for flat-panel displays, making them very inexpensive. Optical testing consisted of hoisting the lens in a vertical position (see photo), illuminating it with monochromatic light from a laser and observing the focal spot produced by the lens. We successfully tested the lens at 405-, 532-, and 670-nm wavelengths. The focal spot size and shape are dominated by the aberrations inherent in the glass panels and the reduced precision in the alignment of the panels. Both these limitations will be overcome in the future.

(S. Dixit, R. Hyde)

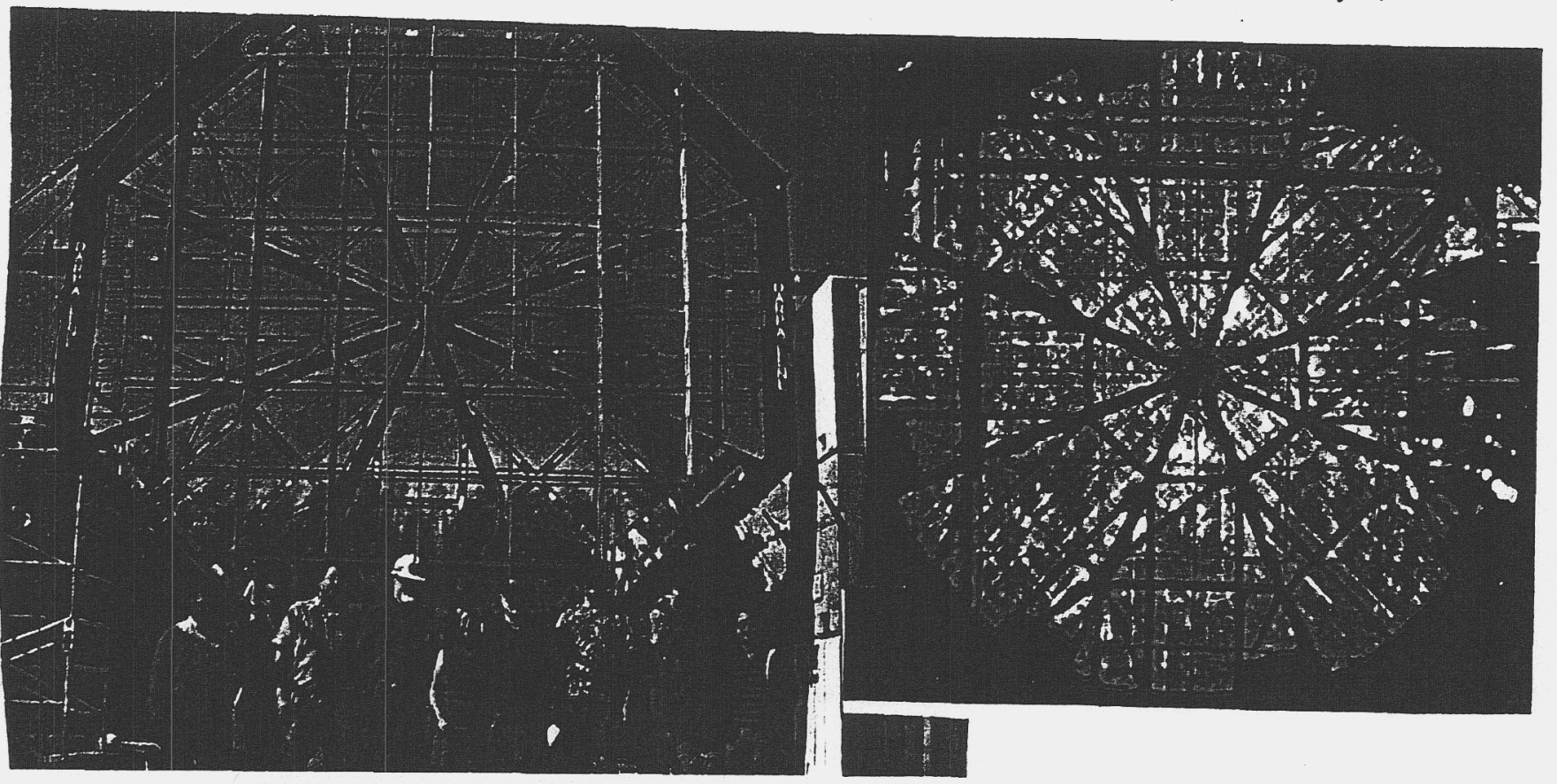




\section{Advanced Solid-State Lasers and Components}

LS\&T provided development and expertise for a number of DOE and DOD projects. These include developing advanced $100-\mathrm{kW}$-class high-power diode-laser arrays for the Mercury laser and Solid-State Heat Capacity Laser (SSHCL) projects, developing advanced fiber lasers for petawatt and laser guide star applications, activating $x$-ray ablation and damage experiments to evaluate IFE optics, and completing construction and beginning activation of the Mercury laser. Mercury is a kW-class, diode-pumped, solid-state laser (DPSSL), intended to serve as driver for highenergy-density physics and inertial fusion energy.

(S. Payne)

\section{Mercury Laser: a Diode-Pumped, Gas- Cooled, Solid-State Slab Laser for Inertial Fusion Energy (IFE)}

Diode-pumped solid-state lasers are one of four driver technologies being considered for inertial fusion energy power plants. Each driver development project, including solid-state laser, heavy ions, Z-pinch and gas laser $(\mathrm{KrF})$, is currently focused on demonstrating several proof-of-principle design concepts. The Mercury laser is being activated and will test key technologies including high-power laser diodes, crystals, and gas cooling within an architecture that is modular and scalable to multi-kilojoule apertures with minimal beam line reengineering.
The design requirements of the laser are to operate at $10 \mathrm{~Hz}$ and $100 \mathrm{~J} /$ shot within a $10 \%$ efficiency envelope. To meet this goal, we explored numerous gain materials and architectural options to best fit the design space of a fusion energy class laser. $\mathrm{Yb}^{3+}: \mathrm{Sr}_{5}\left(\mathrm{PO}_{4}\right)_{3} \mathrm{~F}$ or Yb:S-FAP was chosen as the gain medium based on its long energy storage lifetime, moderately high cross section, and good thermal conductivity. The primary challenge has been in growing large aperture slabs of $4 \times 6 \mathrm{~cm}^{2}$ in area. However, the growth techniques employed to eliminate or reduce the crystalline defects produced boules that were difficult to cut due to the high thermal gradients required during the growth process. Using stress models developed in conjunction with a research group at UC Davis, we chose an off-center cut geometry with a high-pressure water jet to overcome the high probability of boule-fracture during fabrication. This method has proven successful and five SFAP slabs have been mounted onto the first amplifier. Four additional slabs are in fabrication and seven crystalline boules are waiting to be cut. The slabs are mounted onto aerodynamic aluminum structures called "vanes" that are separated by 1 -mm helium gas cooling channels. Recent measurements of $\mathrm{Yb}: S-F A P$ indicate damage thresholds of greater than $50 \mathrm{~J} / \mathrm{cm}^{2}$.

The laser diodes at $900 \mathrm{~nm}$ are precision mounted onto etched silicon heatsinks with microlenses to help reduce the light divergence, and then secured onto large copper blocks or arrays that provide the cooling and electrical

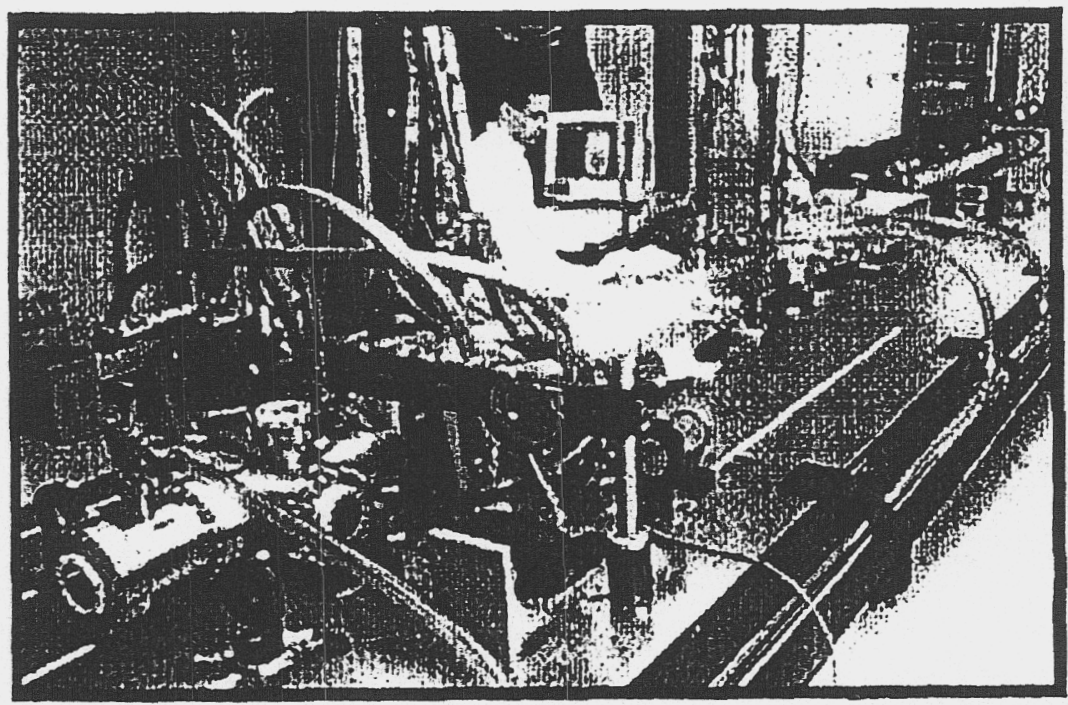

\section{$160 \mathrm{~kW}$ diode back plane}

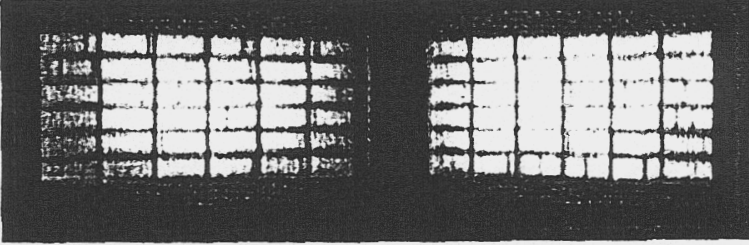

100 W Pockels Cell

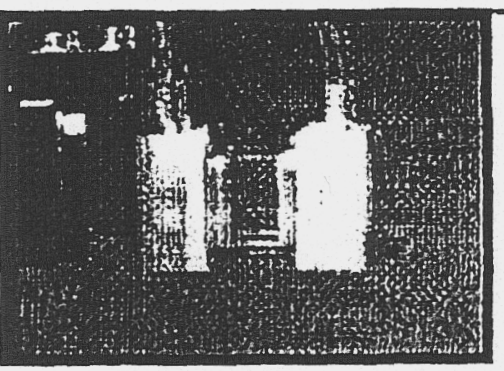


power to the diodes. Each amplifier assembly is pumped by four $80-\mathrm{kW}$ diode arrays. Four arrays have already been built and are currently being exercised in the system. Together they have accumulated over $10^{5}$ shots. Several sets of diode array backplanes have been qualified and will be used for the second amplifier assembly. The diode array light is guided to the amplifier through multiple reflections within a hollow lens duct and homogenizer.

The amplifier was assembled with five Yb:S-FAP slabs and two undoped glass slabs. The undoped slabs serve as placeholders in the amplifier until the remaining S-FAP slabs can be fabricated. With $200 \mathrm{~mJ}$ of injected energy, we were able to extract up to $31.6 \mathrm{~J}$ of energy at $1.047 \mu \mathrm{m}$ in single-shot mode. At $10 \mathrm{~Hz}$, we measured an average energy of $20.6 \mathrm{~J}$. Wavefront data was taken for static (no diode pumping) and full pumping conditions. The static wavefront data show 2.7 waves of peak distortion due to bonding phase discontinuities incurred during the fabrication process. New fabrication techniques are being tested to eliminate these distortions. In the interim, a static conjugate phase plate, using the wet-etch technology developed by LS\&T, was used to correct for the distortion when placed directly adjacent to the amplifier assembly. We measured a factor of three improvement in the energy within a diffraction-limited spot for unpumped conditions. The conjugate phase plate will be used in the system until the slabs can be replaced with ones with higher optical quality.

The overall architecture employs an offaxis, four-pass beam layout. Relay imaging of the laser beam with telescopes between the amplifiers helps to greatly reduce the intensity modulation at the crystalline amplifiers. A100 $\mathrm{W}$, large aperture birefringence-compensated Pockels cell is used after two passes at low fluence $\left(<1 \mathrm{~J} / \mathrm{cm}^{2}\right)$ to help suppress parasitic beams. The laser system, diode laser power conditioning, and utilities are computer controlled. A full suite of sensor packages is used to diagnose the beam after each pass. A dark field diagnostic allows detection of damage on every shot such that when a damage site $>100-\mu \mathrm{m}$ is detected in the dark field image, the laser is shut down immediately. Future plans include installation of the remaining two S-FAP slabs in the first amplifier head, followed by single amplifier experiments and then activation of the second amplifier. When complete, Mercury laser will yield up to $100 \mathrm{~J}$ /pulse at $10 \mathrm{~Hz}$.

(C. Bibeau, A. Bayramian)
Fabrication of MW-Class Laser Diode Arrays for Optical Pumping of Lasers

LS\&T continues to develop diode laser arrays packaging technologies for optical pumping of $\mathrm{kW}$ class and heat capacity lasers. In 2002, we fabricated two 42-kW peak-power packaged arrays for the solid-state heat capacity laser (SSHCL) and eight $80-\mathrm{kW}$ packaged arrays (backplanes) for Mercury laser projects.

To imagine $100-\mathrm{kW}$ diode arrays becoming a common reality, two key elements of the technology need to fall into place: (1) highperformance, reliable diode bars and (2) heatsinks that offer excellent thermal management and precision diode bar mounting. Rapid cooling of diode laser arrays is achieved using silicon microchannels. Photolithography and etching techniques are applied to produce tens of thousands of 30-micron-wide channels in silicon substrates. Water flowing through these microchannels aggressively cools the laser diode bars, which are mounted on the silicon at location less than 200 microns from the channels. By combining 10 diode bars onto a single heatsink, a 10-bar package, referenced as a tile, serves as the unit cell from which large twodimensional diode arrays can be built up through tiling. We have recently completed packaging of several diode arrays. Each package is capable of generating in excess of $40 \mathrm{~kW}$ of optical power. Noteworthy is that the brightness (i.e., W/cm ${ }^{2}$ str) of the array is extremely high, by virtue of the compact tiling of the heatsinks and the precision placement of 10 -element microlens arrays on each tile.

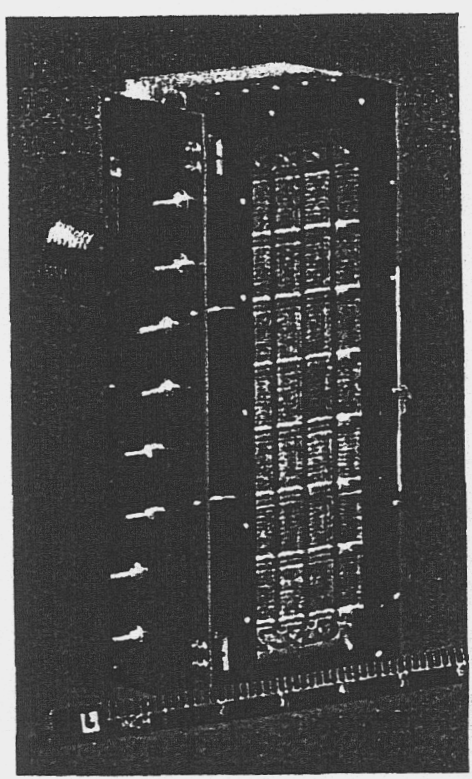


The individual tiles that are used to make up these large arrays are called SiMMs for Silicon Monolithic Microchannels. The considerations that drove the SiMMs package design were ease of fabrication and the ability to construct large laser diode arrays with output power capabilities of 10 to $100 \mathrm{~kW}$. Of paramount importance in the design of the SiMMs package was the requirement that the same aggressive heat removal capability that characterized our original rack-and-stack silicon microchannel-cooled package be preserved in the SiMMs. This requirement was met by incorporating microchannels into the silicon directly below the location of the attached laser diode bars. The SiMMs design has a very tight thermal circuit with only 177 microns of silicon separating the heat-generating laser diode bars and the microchannel fins that define the cooling channels.

Since almost all applications at LLNL now require microlensed arrays, the development of an easy and efficient microlensing attachment technology was an important consideration during the design phase of the SiMMs package. Easy and efficient microlens attachment translated into a requirement that an entire SiMMs package be microlensed in a single step. Single-step microlensing of an entire tile eliminates the production steps necessary to individually attach a microlens to each diode bar as we do in our rack-and-stack package. From our previous experience with the rack-and-stack package, we know that microlenses must be placed with a positional tolerance of a few microns relative to the diode bar emitter facets to achieve good optical performance. This implies that the single-step microlensing of an entire SiMMs tile will require the individual diode bars within a tile be positioned relative to one another with at least the same few-micron accuracy.

The precision placement of the laser diode bars on the SiMMs package is accomplished by using a V-groove technology on the front surface of the package. These Vgrooves are generated using the same etching technology that is used to fabricate the microchannels into the backside of the silicon. The emission direction of the diodes at $55^{\circ}$ to the normal of the SiMMs front face is due to the orientation of the $\mathrm{V}$-grooves that serve as pads for the laser diode bars. With the diode bars precision located, the microlenses can be held in precision frames fabricated in the form of silicon runners using the same anisotropic etching technology that is used to fabricate the V- grooves and the microchannels. Shaped cylindrical microlenses are preloaded and glued into these silicon runners, forming a ladder-like structure consisting of 10 lenses as shown in the photo. The entire 10-lens assembly is then attached to the SiMMs package in a single step. The microlens array serves to collimate the fast axis radiation of the laser diode bars from its original $30^{\circ}$ divergence angle into a collimated beam with a divergence angle of $<1^{\circ}$.

The backing glass blocks of the SiMMs package are designed to sit side by side on a backplane structure containing cooling water source and drains. The package is specifically designed for close packing to give high effective fill factors. The array shown above has an effective fill fraction of 0.7 . This pump module generates $42 \mathrm{~kW}$ of pump radiation at an effective irradiance, including the hit from the less than unity fill of the backplane, of $1 \mathrm{~kW} / \mathrm{cm}^{2}$ when viewed in the direction of the emitted radiation. The SiMMs package represents a significant breakthrough in high-power diodearray packaging technology and enables the scaling of power from 2-D diode arrays to 100 $\mathrm{kW}$ or larger with extremely high brightness. A total of $840 \mathrm{~kW}$ arrays have been installed for the heat-capacity laser program. These are the most powerful average-power diode arrays in existence.

(R. Beach, B. Freitas)
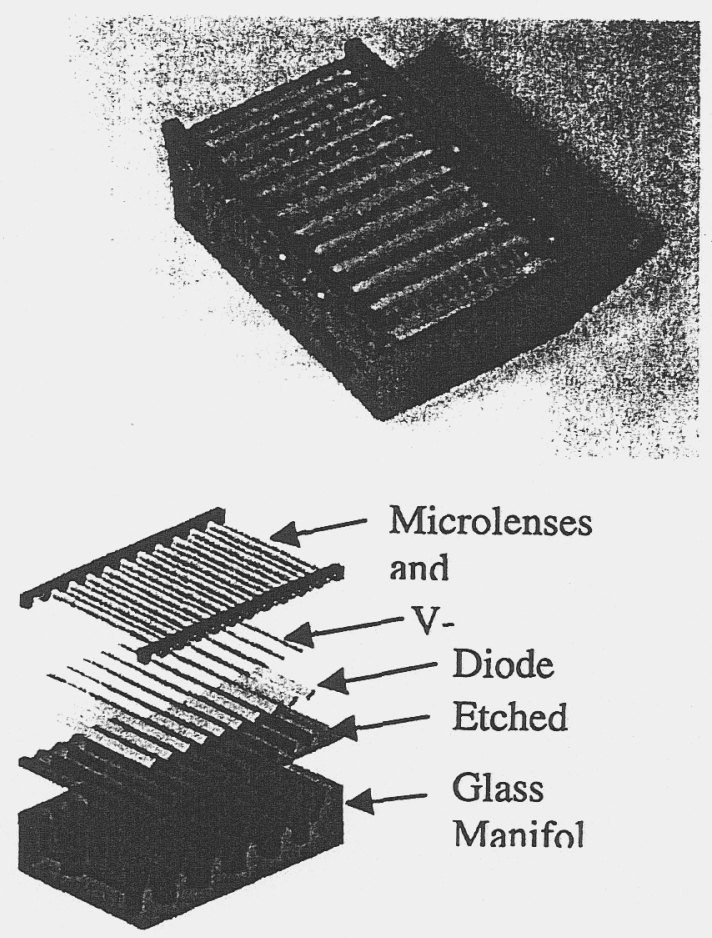


\section{Advanced Fiber Lasers for Laser Guide Star Applications and Petawatt Laser Front Ends}

Since their "rediscovery" by the University of Southampton in 1985, fiber lasers and amplifiers have steadily increased in importance and output power. They are popular solutions to many problems because they typically have high wall-plug efficiency and are easy to package into robust, reliable turnkey devices that virtually anyone can operate. A cursory analysis of fiber laser development history shows that its average output has been increasing by a factor of 10 every 3 years for roughly the last 18 years and is now reaching output powers of interest to DOE and DoD applications.

Two advanced fiber lasers were investigated by LS\&T in 2002: a high-power 938-nm cladding pumped fiber laser to generate 589-nm light for laser guide star application and an ultrastable mode-locked fiber laser as the front end of high-energy petawatt laser system in NIF.

In the laser guide star effort, we are developing a $10-\mathrm{W}$, compact, all-solid-state laser to be operated at $589 \mathrm{~nm}$. Our design is based on sum-frequency mixing of a 10 -W erbium fiber laser (operated at $1583 \mathrm{~nm}$ ) with a 10-W neodymium fiber laser (operated at $938 \mathrm{~nm}$ ) in a periodically poled nonlinear optical material. We have made significant progress this year. A 10 W erbium fiber laser was successfully assembled using a master oscillator power amplifier configuration. To achieve this output, a $3-\mathrm{mW}$ fiber DFB laser is phase modulated to broaden its linewidth and then amplified in a commercial L-band erbium-doped fiber amplifier.

A cladding pumped neodymium fiber laser was developed and has generated over $2 \mathrm{~W}$ of output power. Operation of neodymium-doped glass at $938 \mathrm{~nm}$ works best in a nearly pure silica glass, which limits the maximum doping concentration of the neodymium. Fiber lasers are particularly well suited to this application because they are a natural medium for achieving long interaction lengths at high power density. Physics relating to the operation of neodymium at $938 \mathrm{~nm}$ were investigated including excitedstate quenching as a function of concentration, stimulated Brillouin scattering, ground state absorption, and competition from the 1088-nm laser line. The fiber offers a natural mechanism for suppressing light at longer wavelengths as the waveguide is subject to a wavelengthdependent bend loss. By winding the fiber on an appropriately sized mandrel, we were able to create a distributed filter in the gain medium to suppress 1088-nm light and pass 938-nm light. We plan to scale the 2-W 938-nm fiber amplifier to $10-\mathrm{W}$ level in the near future. First light of $589-\mathrm{nm}$ laser was generated by frequencymixing of outputs from these two fiber lasers (at $1583 \mathrm{~nm}$ and $938 \mathrm{~nm}$ ) in a 5-cm-long periodically poled lithium niobate (PPLN) crystal. We plan to scale the $589-\mathrm{nm}$ output to 10-W level in 2003 for laser guide star application.

We are also developing an advanced fiber laser to be used as the front end for a highenergy petawatt (HEPW) laser system. Our goal is to generate an all-fiber laser source of $<200$-fs pulses that can be stretched to $3 \mathrm{~ns}$ and amplified to output energies to $10 \mathrm{~mJ}$ completely within the optical fiber. The effort is divided into three tasks that include (1) construction of the fiber laser mode-locked master oscillator, (2) investigation of a chirped fiber Bragg grating for pulse stretching, and (3) demonstration of a large mode-area optical fiber amplifier, with a specialty refractive index profile designed to
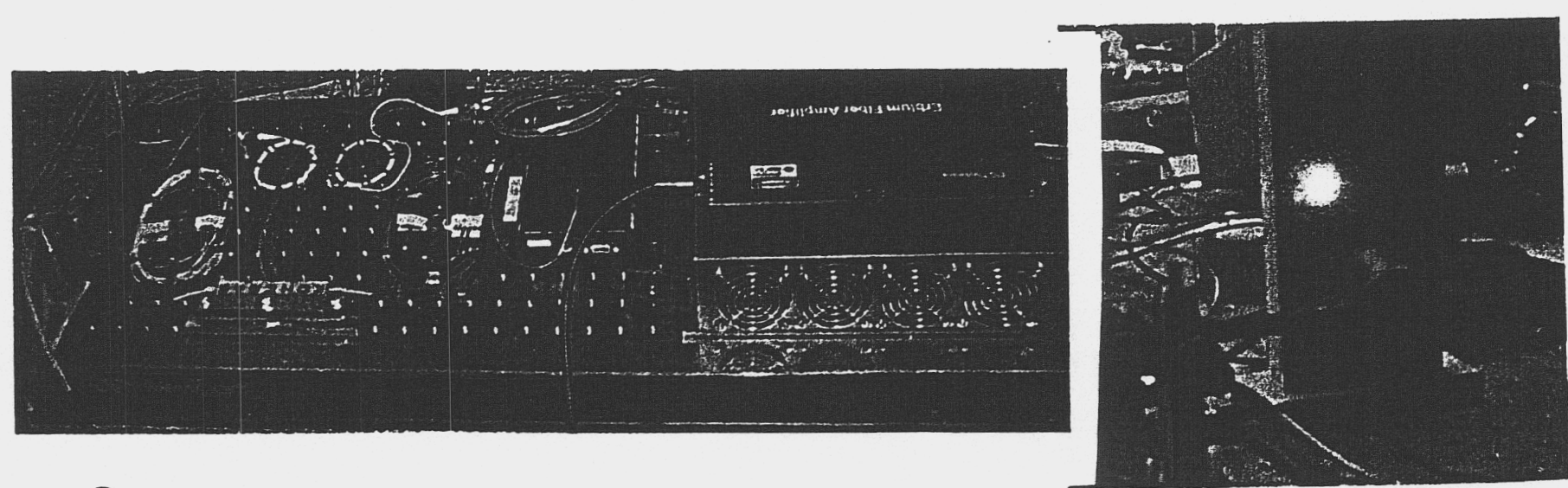
amplify and transmit pulses with up to 2.5 times the energy of optical fiber amplifiers with similar core sizes and beam qualities. We have constructed a working prototype of the modelocked fiber oscillator. We have also designed and procured large-magnitude chirped fiber Bragg gratings for multi-ns pulse stretching. We are continuously investigating the large flattened mode fiber amplifier, with the goal of amplifying laser pulses to energies $>10 \mathrm{~mJ}$ for HEPW on NIF.

\section{(J. Dawson)}

\section{$X-R a y$ Ablation and Neutron Damage Experiments on Optical Materials for IFE}

Under the support of the Defense Program's High Average Power Laser Program, LS\&T is building a facility, named XAPPER, for $x$-ray ablation and damage experiment. The primary objective of this experiment is to study pulsed $x$-ray damage production in candidate inertial fusion energy (IFE) chamber wall (tungsten and carbon composites) and final optic (aluminum and dielectric mirrors, as well as fused silica lenses) materials. XAPPER is also of interest to the heavy-ion fusion program for liquid ablation/condensation studies.

XAPPER (built by PLEX LLC of Brookline, Massachusetts) uses a star pinch to produce soft $\mathrm{x}$-rays. The star pinch is driven by current supplied by six thyratrons that could be switched at $10 \mathrm{~Hz}$ or higher. An ellipsoidal condensing optic is used to collect $\mathrm{X}$-rays and bring them to focus on the sample. When operated with xenon, XAPPER will provide $113-\mathrm{eV} x$-rays at fluences of $\geq 7 \mathrm{~J} / \mathrm{cm}^{2}$. For operation with nitrogen, the peak fluence falls to $-0.25 \mathrm{~J} / \mathrm{cm}^{2}$, but the $\mathrm{X}$-ray energy increases to $430 \mathrm{eV}$. Use of the condensing optic not only allows for higher fluences, it also greatly reduces the debris loading on the sample. Several million pulses of $\mathrm{x}$-rays can be generated before minor maintenance is required. With $x$-ray fluence of several $\mathrm{J} / \mathrm{cm}^{2}$ and the short mean free path of the soft $x$-rays, XAPPER is capable of providing $x$ ray doses that exceed those expected in laser-IFE power plants. Current diagnostics include a photodiode, a vacuum calorimeter, and an extreme ultraviolet (EUV) spectrometer.

To qualify optical materials for IFE, we have performed a series of radiation experiments on fused silica. The survivability of the final optic, which must sit in the line of sight of highenergy neutrons and gamma rays, is a key issue

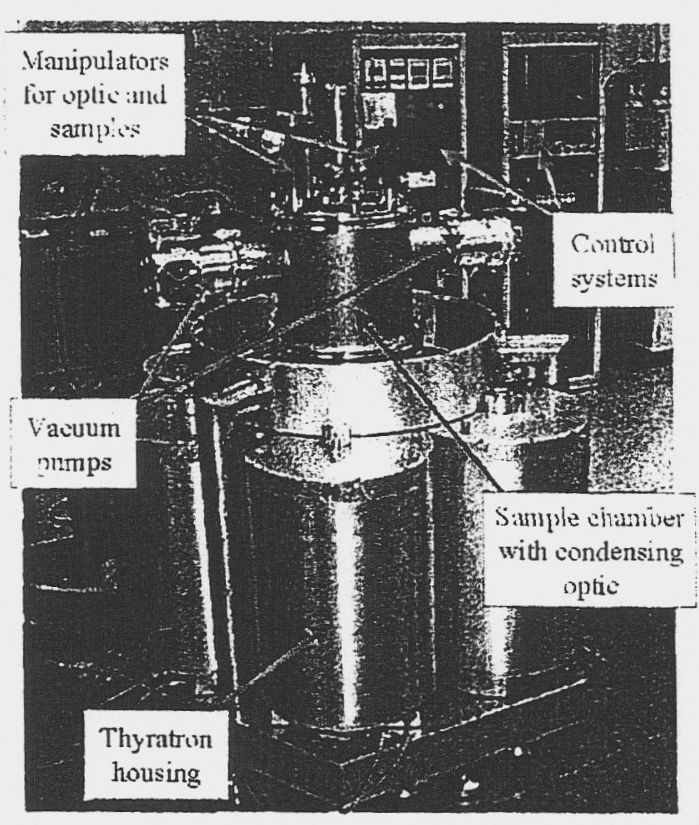

for any laser-driven IFE concept. Previous work has concentrated on the use of reflective optics. We have introduced and analyzed the use of a transmissive final optic for the IFE application. Neutron damage experiments have been conducted at a range of doses and dose rates, including those comparable to the conditions at the IFE final optic. The experimental work, in conjunction with detailed analysis, suggests that a thin ( $<1 \mathrm{~mm}$ ), fused silica Fresnel lens, such as those created for the Eyeglass Project, may be an attractive option when used at a wavelength of $351 \mathrm{~nm}$. Our measurements and molecular dynamics simulations provide convincing evidence that the radiation damage, which leads to optical absorption, not only saturates but that a "radiation annealing" effect is observed. Neutron-induced optical absorption equilibrates at $\sim 5 \%$ for a $500-\mu \mathrm{m}$-thick fused silica Fresnel lens operated at $300^{\circ} \mathrm{C}$. By intentionally operating the optic at an elevated temperature of $500^{\circ} \mathrm{C}$, the optical absorption would be reduced to only $0.6 \%$.

(J. Latkowski)

\section{Advanced High-Power Lasers for High- Energy Density Plasma Physics Experiments}

Under the support of the DOE's NNSA/NV Cooperative Agreement, we are developing a $20 \mathrm{TW} / 20 \mathrm{~J}$ laser at the Nevada Terawatt Facility of the University of Nevada at Reno to perform high-energy-density plasma physics experiments. The purpose of this laser system is to develop proton and $\mathrm{x}$-ray 
radiography backlighting techniques, to study plasmas in z-pinches and magnetically-insulated transmission lines, and to study deposition of energy from high-intensity laser pulses into plasmas. The performance requirements of this laser are: peak irradiance on target $>10^{19} \mathrm{~W} / \mathrm{cm}^{2}$, pre-pulse irradiance $<10^{11} \mathrm{~W} / \mathrm{cm}^{2}$, pointing accuracy of $\pm 25 \mu \mathrm{m}$, timing jitter relative to the Zebra z-pinch facility of $<10 \mathrm{~ns}$ and shot rate $>$ $2 / \mathrm{hr}$. Major efforts were focused on conceptual design of the laser system, which uses an optical parametric chirped-pulse amplifier (OPCPA) for pre-amplification, and phosphate $\mathrm{Nd}$ :glass rod amplifiers for final amplification. A modelocked Nd:glass oscillator is used to generate 2 $\mathrm{nJ}, 250 \mathrm{fs}$-long pulses which are subsequently expanded to $1.5 \mathrm{~ns}$ duration using a grating stretcher. Two optical parametric chirped-pulse amplifier (OPCPA) stages will be used to amplify the pulse to $70 \mathrm{~mJ}$. Two double-passed Nd:glass rod amplifiers will provide the final amplification to $30 \mathrm{~J}$. After reflective gratings recompress the pulse to $<1 \mathrm{ps}$, an $\mathrm{f} / 3$ off-axis parabolic mirror focuses the beam to a $\sim 10 \mu \mathrm{m}$ spot. Faraday isolators is used to protect the laser chain from damage. Analysis shows our design meets energy requirements with acceptable nonlinear phase shift and damage margins. The laser system activation is scheduled in 2004.

LS\&T is also collaborating with the Physics and Advanced Technologies Directorate (PAT) in an upgrade of the JANUS system to achieve $1 \mathrm{~kJ}$ in each of its 2 beam lines. JANUS is a high energy Nd:glass laser system operated by PAT to perform high-energy density experiments. The upgraded laser will enable significant new experimental capabilities, including pressures of $20 \mathrm{Mbar}$, compression to 2-3 times solid density, electron temperatures of $>10 \mathrm{keV}$, and large area shock waves. Major effort was focused on the design of the highenergy amplifiers and beam transport periscopes. Our design codes verified that $1 \mathrm{~kJ}$ laser energy could be achieved at the pulse durätion of $3 \mathrm{~ns}$, using rod amplifiers, and $10 \mathrm{~cm}$ and $15 \mathrm{~cm}$ phosphate glass disk amplifiers. The frequency conversion efficiency is expected to be $>70 \%$. The installation of the upgraded beamline is scheduled for 2003. When completed, it will provide significant support capability for NIF, including testing and calibration of diagnostics and training new physicists and engineers to perform high-energy density experiments.

(A. Erlandson, J. Caird)

\section{Solid-State Heat Capacity Laser for Defense}

Under the support of the U.S. Army's Space and Missile Defense Command, and in collaboration with industrial partners including Decade Optical Systems (DOS), Northrop/Grumman, and others, we are developing high-average-power (100 kW-class), diode-pumped, solid-state heat capacity laser (SSHCL) technology for applications in tactical short-range air defense missions. As a proof-ofprinciple, we have built and delivered a $10 \mathrm{~kW}$ class (13 kW actual) heat capacity laser to White Sands Missile Range. This laser employed flashlamp pumping of the laser media and $\mathrm{Nd}$ :glass laser slabs. In order to advance to a 100 $\mathrm{kW}$-class laser, one needs to transition to laserdiode pumping (for increased power and

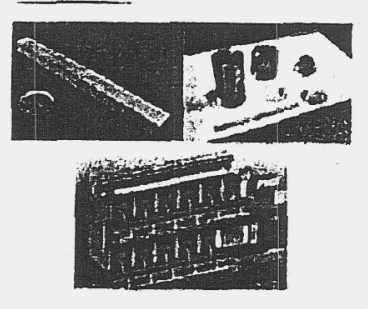

$100 \mathrm{~kW}$ Solid-State Heat-Capacity Laser System

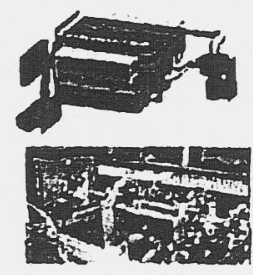

$100 \mathrm{~kW}$ Engineering Model mounted on a Hybrid-Electric HMMWV

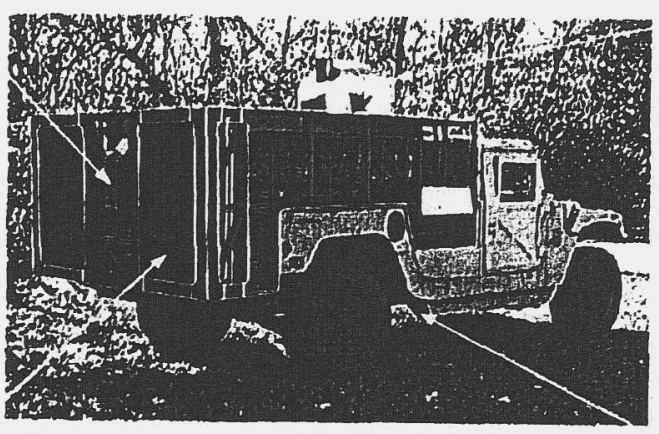

Thermal

Management

System

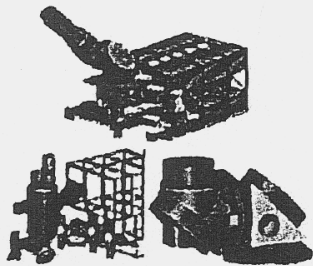

Targeting and Beam Control

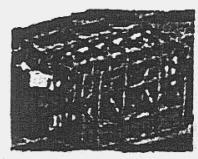

Power

Supply

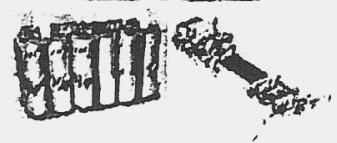


efficiency), and crystalline laser media (for better thermal characteristics). The baseline design for such a laser uses Nd:doped gallium gadolinium garnet (GGG) as the laser medium and laserdiode pumping at $808 \mathrm{~nm}$.

The technical and engineering basis required for SSHCL were established. A mobile, compact, lightweight laser system capable of being deployed on a hybrid electric vehicle is currently under development. When complete, this laser system would be capable of defending against short-range rockets, guided missiles, artillery and mortar fire, and unmanned aerial vehicles (UAV). Power for both the vehicle and laser system would come from the vehicle's diesel generator and onboard high-energy lithium ion batteries.

(B. Dane)

\section{High-Average-Power Pulsed Laser- Material Interaction}

The flashlamp-pumped heat-capacity demonstrator laser was delivered to the High Energy Laser System Test Facility (HELSTF) at the White Sands Missile Range in August 2001 and was operated in support of material interaction testing. In its present configuration using a stable optical resonator, it is capable of generating laser pulse energies between 500 and $1000 \mathrm{~J} / \mathrm{pulse}$ and an average power of up to 13 $\mathrm{kW}$ for 10 -s burst duration. This combination of high pulse energy at high average power has never been achieved from a solid-state laser system. In 2002, an experimental program was undertaken to study the interaction between this beam and target samples made of aluminum and steel. Detailed numerical modeling was developed to guide the experiments and to help interpret the results. We investigated a number of significant interaction effects that are unique to the high-energy, pulsed-beam format. Initial test data indicate that laser-material interactions under high peak irradiance conditions using a small focusing spot result in rapid penetration due to hydrodynamic forces driven by vaporization. Due to transient surface temperature rise during each laser pulse,
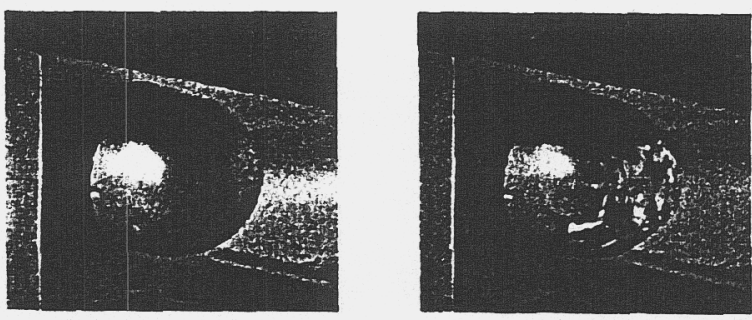

increased heating over that achieved from a $\mathrm{CW}$ laser of the same average power is observed. High peak surface temperatures during each laser pulse also drive the combustion of the metal target under high-speed air flow conditions, providing additional energy deposition and heating.

In 2002, we had the opportunity to irradiate an antitank guided munition (ATGM) with the 10-W SSHCL. A video sequence of the actual ATGM crushing is shown below. The crush fuse on the ATGM is made from an aluminum alloy. The mechanism used to trigger the ATGM fuse was straightforward. The nose of the ATGM is a crush switch consisting of inner and outer domes. When heated by the laser, the fuse is weakened and collapses under the aerodynamic air flow load. When the two domes contact, the fuse is activated. The total fluence required to compromise the ATGM was 702 $\mathrm{J} / \mathrm{cm}^{2}$, a figure almost 10 times less than the value that has been used in the battlefield simulations using an existing Directed Energy (DE) laser system.

(S. Fochs)

\section{Adaptive Resonator Control for High Beam Quality Operation}

Low divergence is necessary to propagate a high-average beam from an SSHCL to target. To do this, the relatively small thermally induced optical distortions in the heatcapacity amplifier of the laser system must be corrected. An unstable resonator that incorporates an intracavity deformable mirror has been incorporated to make these corrections. LS\&T successfully demonstrated lasing from an SSHCL with wavefront correction using the 3slab flashlamp-pumped testbed amplifier. A new mirror, built to tighter specifications was developed to achieve wavefront correction at full average power. A complete understanding of the impact of small-scale structure (ripple) of only $25 \mathrm{~nm} \mathrm{rms}$ is now in hand and a combination of laboratory measurements and numerical simulation has validated a new mirror design. We have recently demonstrated $<1.5$ times the
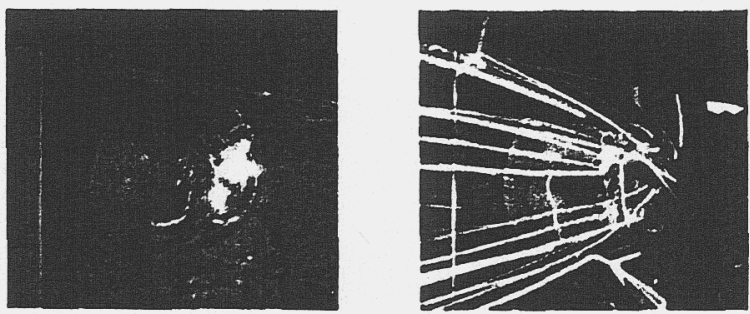
diffraction-limited divergence in active lasing experiments using a full-scale resonator and 3slab flashlamp-pumped amplifier with the original deformable mirror. The full $10-\mathrm{kW}$ amplifier head has now been retumed from HELSTF to LLNL where it is being integrated with the adaptive resonator to demonstrate highbeam-quality operation for a 10 -second burst at full power.

(B. Dane)

\section{Diode-Pumped 3-Slab Nd:GGG Amplifier}

The scaling from the $10-\mathrm{kW}$ flashlamppumped demonstrator laser to $100-\mathrm{kW}$ will use diode-pumped neodymium-doped gadolinium gallium garnet (Nd:GGG) laser. LS\&T is building a 3-slab amplifier testbed as the first conceptual submodule of the $100-\mathrm{kW}$ laser design. The 3-slab testbed will use three fullaperture Nd:GGG crystals. To establish a solid technical basis for such a laser, we are also developing a half-scale testbed that utilizes a single Nd:GGG slab with an active region measuring $5 \times 10 \mathrm{~cm}^{2}$ in size and pumped by four half-size laser diode arrays. The diode arrays consist of 28 SiMMs (Silicon Monolithic Microchannels) "tiles" arranged in a $4 \times 7$ configuration that produce an average optical power of $4.2 \mathrm{~kW} /$ array. The pulse width is nominally $500 \mu \mathrm{s}$. We performed a series of experiments to verify the design of the $100-\mathrm{kW}$ SSHCL system with respect to pulse energy, efficiency, and run time. Optical gain coefficient, pump uniformity, output wavefront, and temperature rise in the lasing medium were also measured to benchmark the temperaturedependent energetic model.

Using a stable resonator with low output coupling, we obtained $\mathrm{kW}$ output from the half-scale system. At $200 \mathrm{~Hz}$, over a tensecond run, we achieved an average power of 2.8 $\mathrm{kW}$ with an initial output in excess of $3 \mathrm{~kW}$. The

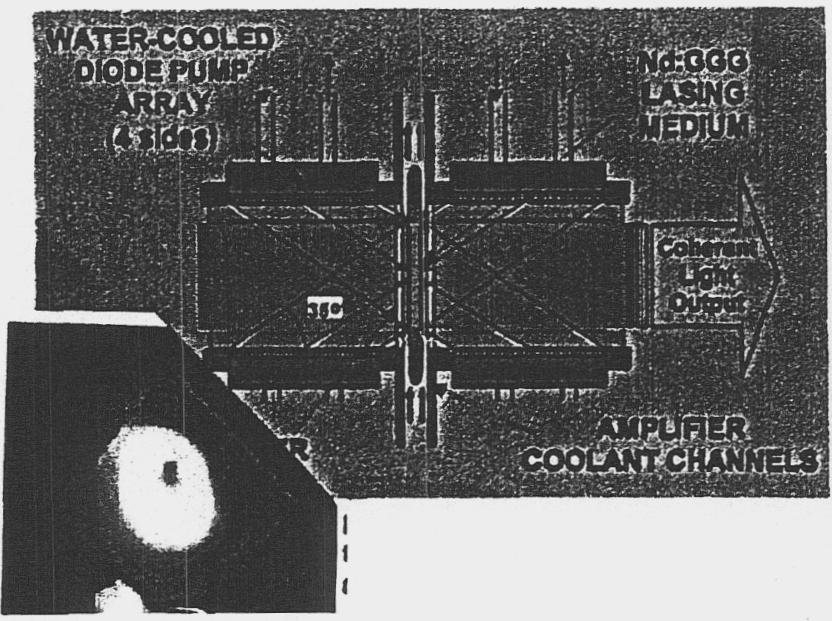

average diode-array optical pump power used for this experiment was $13.3 \mathrm{~kW}$, and the overall diode electrical efficiency was $45 \%$. Initial laser testing has demonstrated $9 \%$ electrical-to-optical efficiency, a figure very near the $10 \%$ goal designed for the $100-\mathrm{kW}$ mobile laser system.

Using the output beam froin the halfscale system, we were able to penetrate a 1.5mm-thick steel coupon in a matter of a few seconds. The mechanical structure of the 3-slab testbed is nearing completion, and the diodearray thermal management system is fully activated. Six of the required 12 full-scale diode arrays ( $80-\mathrm{kW}$ peak each) manufactured by Armstrong Laser Technology have been tested and qualified. Six additional arrays have been manufactured and are undergoing testing. Fabrication of the three Nd:GGG slabs, including diffusion-bonded edge cladding and optical coating, is complete. We plan to activate the three-slab diode-pumped Nd:GGG testbed in 2003 as a scientific prototype for the $100-\mathrm{kW}$ system to be developed for battlefield defense. It is anticipated that this system will generate an output power in excess of $15 \mathrm{~kW}$-greater than the nine-slab flashlamp-pumped system currently in operation at the Army's HELSTF.

In additiona to SSHCL laser

development, LS\&T has also conducted battlefield simulation using the Joint Conflict and Tactical Simulation (JCATS) code with a goal of parameterizing the target engagement and kill time in order to provide a better understanding of required laser power for an objective weapons system. Past work has laid the experimental and theoretical foundations of SSHCL operation and successfully demonstrated the concept with the flashlamp-pumped Nd:glass laser system. For the first time we have now demonstrated high-efficiency operation of a diode-pumped Nd:GGG SSHCL and are poised to operate the first conceptual submodule of a $100-\mathrm{kW}$ objective laser system.

(M. Rotter, B. Yamamoto)

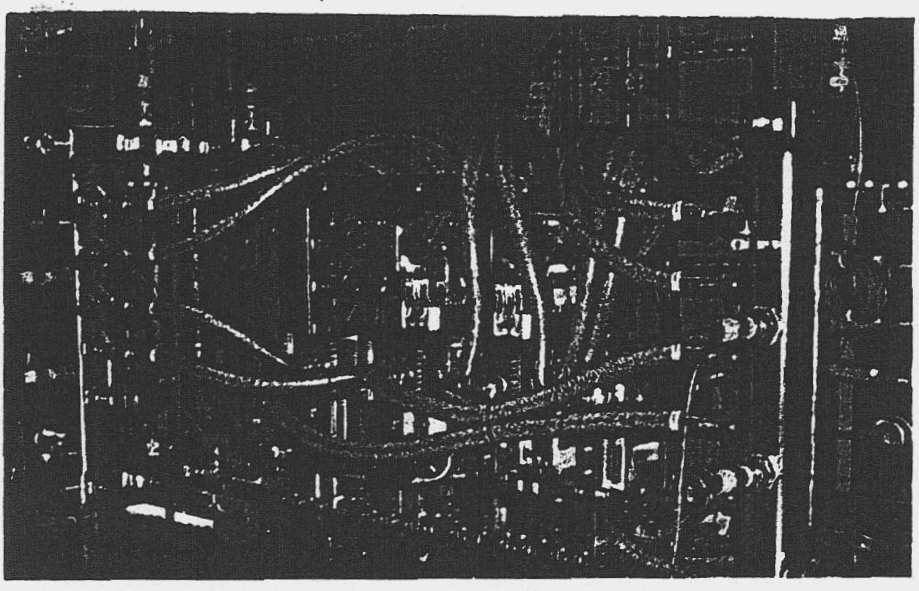




\section{Material Processing Using Pulsed Solid-State Lasers}

Laser peening is an emerging surface treatment technology for metals that places deep residual compressive stress into the surface, greatly improving resistance to fatigue failure and stress corrosion cracking. A laser system appropriate for peening at an industrial level requires an average power in the hundred-watt to kilowatt range, a pulse energy of around 25-to$100 \mathrm{~J}$ per pulse and a pulse duration of 10-to-30 ns. A solid-state laser with such capability was first developed for a DoD mission in 1996 by Drs. Lloyd Hackel and Brent Dane of LLNL. In order to complete the chain required for technology commercialization, we successfully established a Cooperative Research and Development Agreement (CRADA) with the Metal Improvement Company (MIC) of Parnasus, NJ. During the past year, we demonstrated key process performance, supported assembly of several high-rate, production-type laser-peening systems, and MIC began peening of commercial jet engine components. LS\&T is currently developing the laser peening process to improve fatigue and corrosion lifetimes of metals and alloys in several project areas: (1) for DoE's Civilian Radioactive Waste Management System program, Yucca Mountain Project (YMP), to improve the service lifetime of metal canisters designed for long-term disposal of high-level radioactive waste generated by commercial nuclear power plants and government reactors, (2) for the U.S. military to improve the fatigue lifetime and reliability of aircraft structures as well as gears, bearings, and drive trains used in rotorcraft and combat vehicles and other energygeneration equipment in the DoD' $s$ weapon system, (3) for oil and gas exploration and production, to improve the performance and reliability of structural materials. Spin-off technologies also demonstrate that pulse lasers are making an impact on U.S. industry in general; for example, technology like laser peenmarking, which provides an easy way to identify parts, and peenforming, which enables complex contouring of problematic thick metal components such as parts of large aircraft wings. We have recently partnered with the Material Performance Laboratory at the University of California, Davis, to further the development of laser peening.

\section{Laser Peening Improves Corrosion Resistance and Mitigates Tensile Residual Stresses in Alloy 22}

Under the support of Bechtel SAIC, and in collaboration with UC Davis and Metal Improvement Company, we are developing a laser peening process for application to radioactive waste storage. DOE has been charged with designing, constructing and operating a facility at the Yucca Mountain Project's (YMP) Nevada site to permanently store the nation's high-level civilian and military radioactive waste. In addition to the natural barriers at the Nevada site, a number of engineered barriers are being designed to help the facility meet its containment goal. Foremost among these is the waste package, which will be fabricated from Alloy 22, which is one of the materials known to be most highly resistant to corrosion. Our current work has focused on mitigating the potential for stress corrosion cracking (SCC) by eliminating the near-surface tensile residual stresses resulting from welding processes and evaluating the corrosion resistance of laser-peened Alloy 22.

Parametric studies covering many of the laser peening conditions have resulted in an improved treatment plan and the deepest residual stresses we have produced to date. Examples of two-dimensional residual stress fields in peened and unpeened Alloy 22 weldments, measured with the contour method, are shown below.

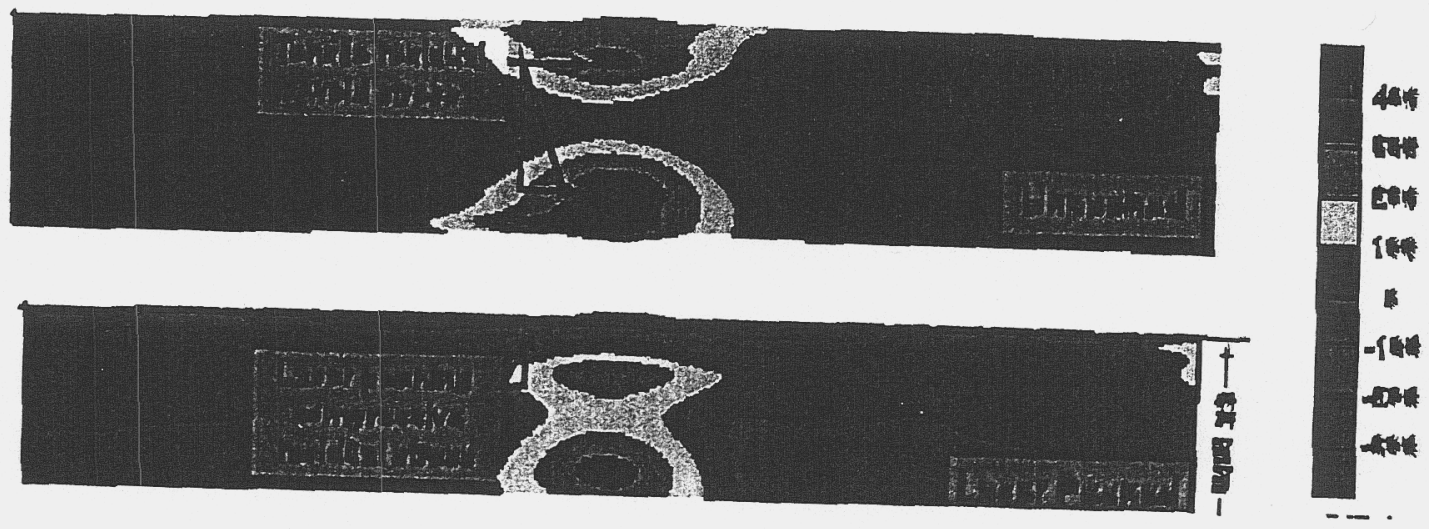


The effects of laser peening are dramatically demonstrated, with the near-surface longitudinal (along the weld) tensile residual stress present in the unpeened plate converted into deep, compressive residual stress with the application of laser peening.

Measurements of residual stress were made for various coupon thickness (from 5 to 35 $\mathrm{mm}$ ). The depth of compressive residual stress appears to increase with coupon thickness. These depths of residual stress, on the order of $20 \%$ of the part thickness for the range tested, far exceed the capabilities of conventional shot peening and other methods used in industry. This deep compressive stress serves to inhibit the process of SCC, which occurs in a tensile stress field. We have also measured the corrosion rate of peened and unpeened Alloy 22 coupons. Preliminary results have shown that laser-peened Alloy 22 will corrode more slowly than Alloy 22 alone. Because of the large depth of residual compressive stress, laser-peened material will continue to inhibit SCC even after several millimeters of material are removed through general corrosion. These beneficial characteristics, in conjunction with its noncontact, "hands off" nature, make laser peening a good candidate for YMP to improve the corrosion resistance and lifetime of storage canisters.

(J. Rankin)

\section{Laser Peening Improves Fatigue Life of Aluminum Components for USAF}

Under the support of the United States Air Force's (USAF's) Aging Landing Gear Life Extension Program, LS\&T is working with UC Davis, the Ogden Air Logistics Center Landing Gear Engineering Branch (OO-ALC/LILE), and Metal Improvement Company, Inc. (MIC) to evaluate the advantages of applying laser peening (LP) to improve the performance of landing gear components for the USAF. Using test coupons made from the same high-strength aluminum alloy (7049-T73) as the trunnion, we systematically studied the effects of laser peening on the fatigue life and SCC characteristics of a trunnion, which is a part of the main landing gear on the USAF's T-38 aircraft. The results clearly show that laser peening can significantly improve the fatigue life of this component and its resistance to SCC.

Since the trunnions are currently treated with a shot peening (SP) treatment, an additional goal of this study was to compare the two surface treatments (LP and SP) and to also investigate the potential effects of applying laser peening to previously shot-peened specimens. Fatigue tests at various load levels near the service stress were performed. All three surface treatment techniques (laser peening $\mathrm{LP}$, shot peening SP, and laser peening on top of the shot peening process $\mathrm{LS}+\mathrm{SP}$ ) increased the fatigue life of the test specimen but the greatest benefits appear to come from the laser peening treatment. Additionally, the benefits of each of the surface treatments increased at longer fatigue lives (lower maximum stress).

Stress corrosion cracking tests were performed on C-ring specimens made from 7075-T6 aluminum. The same surface treatments as above were used for the SCC tests (LP, SP, SP+LP) along with a control case of no surface treatment. The untreated $\mathrm{C}$-ring specimens all showed clear evidence of SCC and cracked in half in the caustic environment after a few days $(11,13$, and 23 days) while none of the treated specimens cracked throughout the entire test duration of 60 days. Although this test was not conclusive in ranking the benefits of LP over SP with regard to SCC, it did clearly show that LP is an effective tool to enhance the SCC performance of untreated specimens.

We are currently performing similar laser peening and fatigue tests on actual T-38 trunnions. Since the fatigue coupons were specifically designed to have a fatigue performance similar to the T-38 trunnion, it is expected that a similar life extension is achievable on the actual part. The estimated cost savings to the USAF (due to a reduction in the replacement costs and maintenance and inspection time) will be on the order of tens of millions of dollars. (A. Demma, A DeWald)

\section{Commercialization of Laser Peening Process and Tool}

While the YMP and USAF development work continues, laser peening was also introduced this past year into commercial production by Metal Improvement Company, our CRADA partner. The laser peening system was deployed to solve an important fatigue failure problem in high-value commercial jet engine components. Since commercial introduction in May 2002, aircraft worth billions of dollars are now in service with laser-peened parts-saving millions of dollars per month in aircraft maintenance costs, millions more in parts replacement costs, and all the while greatly enhancing safety. 


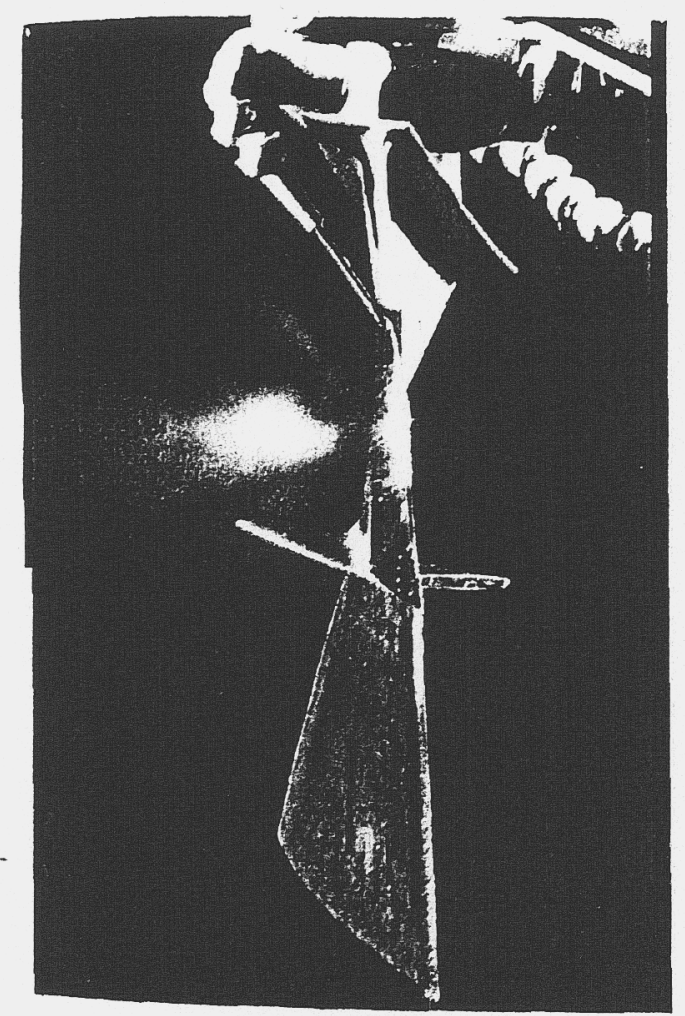

DISCLAIMER

This document was prepared as an account of work sponsored by an agency of the United States Government. Neither the United States Government nor the University of California nor any of their employees, makes any warranty, express or implied, or assumes any legal liability or responsibility for the accuracy, completeness, or usefulness of any information, apparatus, product, or process disclosed, or represents that its use would not infringe privately owned rights. Reference herein to any specific commercial product, process, or service by trade name, trademark, manufacturer, or otherwise, does not necessarily constitute or imply its endorsement, recommendation, or favoring by the United States Government or the University of California. The views and opinions of authors expressed herein do not necessarily state or reflect those of the United States Government or the University of California, and shall not be used for advertising or product endorsement purposes.

This work was performed under the auspices of the U.S. Department of Energy by University of California Lawrence Livermore National Laboratory under Contract W-7405-ENG-48.

This report has been reproduced directly from the best available copy.

Available to DOE and DOE contractors from the

Office of Scientific and Technical Information

P.0. Box 62, Oak Ridge, TN, 37831

Prices available from (615) 576-8401, FTS 626-8401.

Available to the public from the National Technical Information Service, U.S. Department of Commerce, 5285 Port Royal Rd., Springfield, VA 22161 